\title{
Secondary Flow Loss Reduction in a Turbine Cascade with a Linearly Varied Height Streamwise Endwall Fence
}

\author{
Krishna Nandan Kumar and M. Govardhan \\ Thermal Turbomachines Laboratory, Department of Mechanical Engineering, Indian Institute of Technology Madras, \\ Chennai 600 036, India \\ Correspondence should be addressed to M. Govardhan, gova@iitm.ac.in
}

Received 11 September 2010; Revised 2 March 2011; Accepted 21 March 2011

Academic Editor: J.-C. Han

Copyright ( $) 2011$ K. N. Kumar and M. Govardhan. This is an open access article distributed under the Creative Commons Attribution License, which permits unrestricted use, distribution, and reproduction in any medium, provided the original work is properly cited.

\begin{abstract}
The present study attempts to reduce secondary flow losses by application of streamwise endwall fence. After comprehensive analysis on selection of objective function for secondary flow loss reduction, coefficient of secondary kinetic energy (CSKE) is selected as the objective function in this study. A fence whose height varies linearly from the leading edge to the trailing edge and located in the middle of the flow passage produces least CSKE and is the optimum fence. The reduction in CSKE by the optimum fence is $27 \%$ compared to the baseline case. The geometry of the fence is new and is reported for the first time. Idea of this fence comes from the fact that the size of the passage vortex (which is the prime component of secondary flow) increases as it travels downstream, hence the height of fence should vary as the objective of fence is to block the passage vortex from crossing the passage and impinging on suction surface of the blade. Optimum fence reduced overturning and underturning of flow by more than $50 \%$ compared to the baseline case. Magnitude and spanwise penetration of the passage vortex were reduced considerably compared to the baseline case.
\end{abstract}

\section{Introduction}

The term secondary flows refers to the three-dimensional vortical flow structures that develop in blade passages due to high turning of the flow and nonuniform inlet total pressure profiles. Primary flow is the flow which is responsible for the torque generation. Flow which is transverse to the primary flow direction is termed as secondary flow. The boundary layer flow along the endwall contains spanwise velocity gradients. When the boundary layer flow is turned, transverse velocity components are introduced. These secondary flows, created at the endwall and blade junction, extract energy from the fluid which would otherwise be used to rotate the blades or produce thrust. If these secondary flows can be weakened, more energy would be available for torque and thrust production.

Horseshoe vortex, corner vortex, tip vortex, endwall crossflow, and passage vortex are secondary flow components in the cascade. Among these, passage vortex is the primary source of loss. Streamwise endwall fences were employed by
Kawai [1], Moon and Koh [2], and Govardhan et al. [3] for reducing the secondary flow losses in a turbine cascade. Each of the above-mentioned investigations report that the fence of height $1 / 3 \mathrm{rd}$ of the inlet boundary layer thickness when located half a pitch away from the blades results in maximum secondary flow loss reduction. Kawai [1] and Govardhan et al. [3] did experimental investigations, while Moon and Koh [2] did numerical study.

The aerodynamic and endwall heat transfer effects were presented by Camci and Rizzo [4] for six different endwall boundary layer fences in a $90^{\circ}$ square duct. The fences were attached midway between pressure and suction surfaces. The fences reduced the amount of low-energy boundary layer fluid that is convected by the passage vortex to the suction surface. When half height fences were employed, they produced a pair of counterrotating vortices near the fence. On the other hand, full height fences acted as blades and formed two separate passage vortices near the endwall between the fence pressure and suction surfaces and the duct pressure and suction surfaces. 
Flow in a curved channel has no stagnation zone (except a very small zone near to fence), while flow in a turbine comprises of stagnation zone near to the leading edge of blade (also a small zone near the leading edge of fence). Computation of flow using isotropic eddy viscosity turbulence models suffers from a defect called $k-3$ stagnation point anomaly. This anomaly means nearer to the point of stagnation turbulent kinetic energy becomes enormously high. Flow in a curved duct will not have this effect, as it has no major stagnation zone. Chung et al. [5] used a triangularshaped endwall fence in a cascade to prevent the pressureside leg of the horse shoe vortex from washing film-cooling flow off the blade suction surface. Reduced aerodynamic losses and improved cooling performance due to the fence effects on the passage vortex were reported by them. Chung and Simon [6] further investigated endwall boundary layer fences at increased free-stream turbulence levels $(\mathrm{Tu}=10 \%)$ and reported reduced strength passage vortex.

Present study attempts to reduce secondary flow losses by the application of streamwise endwall fences. An innovative design of fence is presented which is found to be more efficient than fence of height $1 / 3$ rd of inlet boundary layer thickness for the present turbine cascade. Selection of the objective function for computational optimization for secondary flow losses is addressed here.

\subsection{Brief Discussion on Objective Function for Secondary Flow} Loss Reduction Techniques. Secondary flow loss reduction in a turbine can be achieved by leading edge modifications, endwall profiling,and by using streamwise endwall fences. For a given turbine cascade and for a particular secondary flow loss reduction technique, there could be many test cases to be tried to determine an optimum. Opting for experimental investigations is certainly a very accurate way of solving the problem but it is highly time-consuming and costly. CFD can predict correctly provided the objective function used in the computation is predicted correctly (at least qualitatively). Few of the quantities which were used as objective function by researchers in numerical study for secondary flow loss reduction are exit flow angle deviation, coefficient of secondary kinetic energy (CSKE), and secondary kinetic energy helicity (SKEH) or combination of these. Ideally the mass averaged total pressure loss coefficient should be taken as objective function, but its prediction by CFD is not accurate [7].

Ingram [8] observed that CFD used in the design of profiled endwalls does not accurately predict loss either in terms of the absolute values of loss or the changes introduced by endwall profiling (qualitative prediction, i.e., increase or decrease). Clearly therefore total pressure loss is not a practical way of optimizing a design. However some way of optimizing the designs needs to be found-even if it is less than ideal. Ingram [8] designed few of the endwall test cases using exit angle deviations as objective function (reduction in exit flow angle deviation). He observed that the optimum endwall generated more losses compared to the baseline case. If exit flow angle is taken as objective function, the reduction in deviation angle could also result from intense corner vortex as well, which in fact will increase the losses.
Therefore, using exit flow angle alone as objective function could be misleading. Ingram et al. [9] designed another set of endwalls using CSKE as the objective function, in which the optimum endwall reduced the losses. Table 1 presents the comparison of CFD and experimental values of total pressure loss coefficient and CSKE for a profiled endwall [8]. The table shows that experimental values of losses and CSKE with profiled endwall are less compared to planar wall. On the other hand, CFD prediction shows increase in total pressure loss coefficient and reduction in CSKE. Work done by Ingram [8], Rose et al. [10], and Harvey et al. [11] demonstrates that using CSKE as objective function for secondary flow loss reduction can be highly successful. Experimental investigations by Kawai [1] and Govardhan et al. [3] (on streamwise endwall fences) and Hartland et al. [12] (on endwall profiling) have shown that a successful design produces a reduction in CSKE and exit flow angle along with a reduction in total pressure loss.

Another quantity called Secondary Kinetic Energy Helicity (SKEH) is also used as an objective function for secondary flow loss reduction. The dot product of SKE (secondary kinetic energy) and helicity $(H)$ is called as SKEH. Helicity is the flux of vorticity:

$$
\text { SKE (secondary kinetic energy) }=\frac{1}{2} \rho\left(c_{\mathrm{sec}}^{2}+c_{r}^{2}\right),
$$

where $c_{r}$ is radial velocity (see [13]) and $c_{\mathrm{sec}}=-c_{m} \sin \overline{\overline{\alpha_{2}}}+$ $c_{u} \cos \overline{\overline{\alpha_{2}}}$

CSKE (coefficient of secondary kinetic energy)

$$
=\frac{\text { SKE }}{1 / 2 \rho{\overline{\overline{c_{2}}}}^{2}},
$$

Helicity $=|c \cdot \omega|$, where $\omega$ is vorticity vector,

$$
\begin{aligned}
& H(\text { nondimensional helicity })=\frac{|c \cdot \omega|}{\overline{\overline{c_{2}}} / c h}, \\
& \text { SKEH (secondary kinetic energy helicity) } \\
& \quad \times(\text { nondimensional })=\text { SKE.H. }
\end{aligned}
$$

The secondary kinetic energy (SKE) at any measuring plane is caused by viscous effects and potential flow (inviscid flow) effect. Because of viscous effect, vortices are generated and vortices cannot be generated in an inviscid flow. In order to consider the SKE associated with vortical components of fluid only (which means excluding SKE caused by potential effects), the dot product of the SKE and helicity is used. Helicity is zero in potential flows (vorticity is zero); therefore, use of SKEH would exclude SKE associated with potential flow. However, SKEH does not exclude the SKE due to the potential field in the regions of vortical flow. This contribution will be small compared with the changes due to the vortical flow. SKEH was used as objective function by Corral and Gisbert [14] and Brennan et al. [15]. Many researchers prefer CSKE as objective function for secondary flow loss reduction. Both CSKE and SKEH can be easily calculated in computations, but in experiments calculation 
TABLE 1: Comparison of $\overline{\overline{\mathrm{CSKE}}}$ and $\overline{\overline{Y_{T}}}$ values (\% of planar values) [8].

\begin{tabular}{|c|c|c|}
\hline & $P 1$ (profiled wall set 1 ) & $P 2$ (profiled wall set 2$)$ \\
\hline \multicolumn{3}{|l|}{ Experimental } \\
\hline$\overline{\overline{\mathrm{CSKE}}}$ reduction & 46.0 & 54.5 \\
\hline$\overline{\overline{Y_{T}}}$ reduction & 21.5 & 30.2 \\
\hline \multicolumn{3}{|l|}{ CFD } \\
\hline$\overline{\overline{\mathrm{CSKE}}}$ reduction & 48.9 & 38.6 \\
\hline$\overline{\overline{Y_{T}}}$ reduction & -1.0 & 6.7 \\
\hline
\end{tabular}

SKEH needs several steps. Even Corral and Gisbert [14] and Brennan et al. [15] who used SKEH as objective function did not present the experimental values. Hence, it is not clear how the experimental and computational SKEH behaved (i.e., whether both showed decreasing/increasing values or one increasing and other decreasing as in case of total pressure loss coefficient) when the flow was subjected to any secondary flow reduction technique. In few investigations combination of above mentioned parameters was taken as objective function. These parameters were weighted and then added. These weighted coefficients may also add further approximations. In most of the studies, where parameters are weighted, no analytical justification is given for their use. Moon and Koh [2] did numerical investigation on streamwise endwall fences. Three different fences were placed in the middle of flow passage, and then optimization was done using the magnitude of passage vortex. When a fence is placed multiple passage vortices and counterrotating vortices are formed. The loss should be decided based on the cumulative effect of all the vortices rather than on passage vortex present near to suction surface of the blade. To some extent secondary kinetic energy can represent the cumulative effect of all the vortices. Because of counterrotating vortices secondary kinetic energy will reduce, but it does not guarantee reduction in losses. Vortex is a source of loss whether it has clockwise-rotation or counter clockwise rotation; hence formation of a counterrotating vortex cannot guarantee reduction in losses. One more effect of counterrotating vortex is reduction in exit flow angle deviations, which is good for next stage of turbine (as incidence losses will decrease for the next stage). Apparently the physics of secondary flow in a turbine needs more exploration. All the parameters suffer from one or other deficiencies. Table 2 presents the objective function used by various researchers for computational optimization in secondary flow loss reduction techniques. For the present study, CSKE is selected as objective function as it is found to be most successful till now.

\section{Approach}

Details of the turbine blades employed in the present investigation are shown in Figure 1. Origin of the computational domain is at the leading edge of the blade (origin $\mathrm{O}$ in Figure 1). Aim of the present study is to optimize the fence height and its location for maximum loss reduction, when it is placed on the endwall. Different fence heights are tested at different locations in streamwise direction on endwall for minimum secondary flow losses. The fence with the optimum height and location will be the one which has least CSKE. Secondary flow loss is directly proportional to CSKE; hence it represents the secondary flow losses (Benner et al. [13]). Here the approach is CFD-based optimization and the objective function for optimization, is CSKE. As the size of the passage vortex is of the order of the inlet boundary layer thickness, the characteristic length scale for fence height has been chosen as inlet boundary layer thickness $(\delta)$. In the present study inlet boundary layer thickness $(\delta)$ is $0.04 \mathrm{~m}$, $(\delta / h=0.1)$. The value was obtained experimentally by Govardhan et al. [3].

\section{Details of Experimental Program}

Govardhan et al. [3] did experimental investigations using a linear cascade tunnel. The tunnel is a blowing type consisting of air supply unit, diffuser, settling chamber, and test section. Seven blades were installed at the end of test section. Fences were attached normal to the endwall and half pitch away from the blades. The inlet total pressure was obtained at $x / a=-1.5$ while downstream survey was conducted at $x / a=1.06$, where $x$ is cascade axial direction and $\mathrm{a}$ is the axial chord. From the experiments it was found that the flow is periodic after a pitchwise distance equal to one blade spacing. Hence in computations only one blade is modeled with fluid interfaces given translational periodicity after a pitchwise distance equal to one blade spacing. Details of blade are given in Figure 1.

A miniaturized five-hole probe having a head diameter of $0.0024 \mathrm{~m}$ was traversed at the exit of the cascade from midspan to the endwall at 26 locations covering more points in the endwall region. For each spanwise location, the probe was traversed in the pitchwise direction at more than 25 locations covering one blade spacing. The probe was used to measure the total pressure, static pressures, and the flow direction in mutually perpendicular planes (yaws and pitch planes). In all the experiments space-chord ratio was maintained constant at 0.79 , and the tip clearance was varied from $\tau / c h=0.0$ to 0.04 , where $\tau$ is tip gap height. The Reynolds number based on the blade chord and outlet mean velocity was maintained at $1.5 \times 10^{5}$.

\section{Numerical Methodology}

For the present investigations fences are fixed normal to the endwall in streamwise direction. The curvature of the 
TABLE 2: Objective functions used for different secondary flow reduction techniques.

\begin{tabular}{|c|c|c|}
\hline Investigator & Secondary flow loss reduction technique & Optimization parameter \\
\hline Brennan et al. [15] & Endwall profiling & SKEH \\
\hline Ingram $[8]$ & Endwall profiling & CSKE \\
\hline $\begin{array}{l}\text { Corral and } \\
\text { Gisbert }[14]\end{array}$ & Endwall profiling & SKEH + an exponential function of inlet swirl angle \\
\hline Bagshaw et al. [16] & Endwall profiling & SKEH \\
\hline Nagel and Baier [17] & Endwall profiling & $\begin{array}{l}\text { Weighted addition of various postprocessor results } \\
\text { (averaged loss accounts for the major part) }\end{array}$ \\
\hline Duden et al. [18] & Endwall and leading edge modification & $\begin{array}{l}\text { Exit flow angle deviation and a secondary flow area with } \\
\text { the least possible distance from the endwall }\end{array}$ \\
\hline Harvey et al. [19] & Endwall profiling & $\begin{array}{l}\text { Cross-passage static pressure gradient on endwall and exit } \\
\text { flow angle deviation }\end{array}$ \\
\hline Pralsner et al. [20] & Endwall profiling & $\begin{array}{l}\text { Total pressure loss coefficient, SKE and TKE (turbulent } \\
\text { kinetic energy) }\end{array}$ \\
\hline Ingram et al. [9] & Endwall profiling & Exit flow angle deviation \\
\hline Germain et al. [21] & Endwall profiling & Combination of total pressure loss coefficient and CSKE \\
\hline Schüpbach et al. [22] & Endwall profiling & CSKE \\
\hline Moon and Koh [2] & Streamwise endwall fence & Stream wise vorticity \\
\hline
\end{tabular}

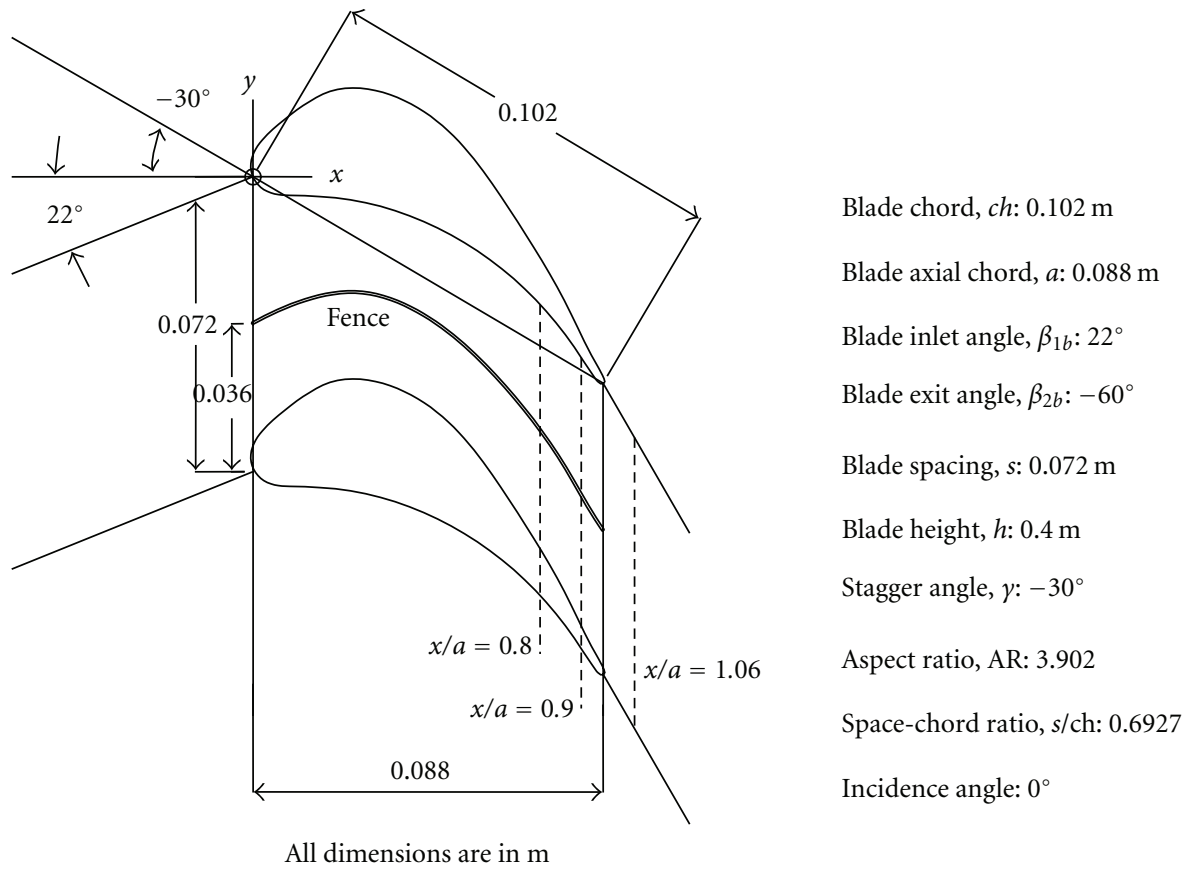

FIgURE 1: Details of cascade.

fence is the same as that of the blade camber line. The fence thickness, $t=0.0007 \mathrm{~m}(t / s=0.00972)$. The thickness of the fence was kept low to prevent blockage of flow. The fence of $0.0007 \mathrm{~m}$ thickness may not withstand high temperature, high pressure, and highly unsteady real engine flow conditions. It could be expected that, in near future, development of new materials may sustain the above mentioned conditions. Numerical simulations were carried out with fences of height, $h_{f} / \delta=0,1 / 6,1 / 3,2 / 3,1.0,4 / 3$. $h_{f} / \delta=0$ refers to the baseline case. Figure 2 shows the mesh and a view of the computational domain. Geometry of the computational domain is created in AUTOCAD. Inlet of domain is at 1.5 chord length upstream of the leading edge. Outlet is at a distance of 3.5 chord length from the trailing edge. The outlet plane is positioned farther as the static pressure $0 \mathrm{~Pa}$ (gauge) is the boundary condition. Grid is made in ICEM CFD. Hexahedral mesh is made for the domain, and around the blade o-grid is employed to get highly orthogonal grid for accurate solution.

According to method of characteristics, flow angle, total pressure, and total temperature are used as boundary conditions at subsonic axial inlet. All solid walls are set with 


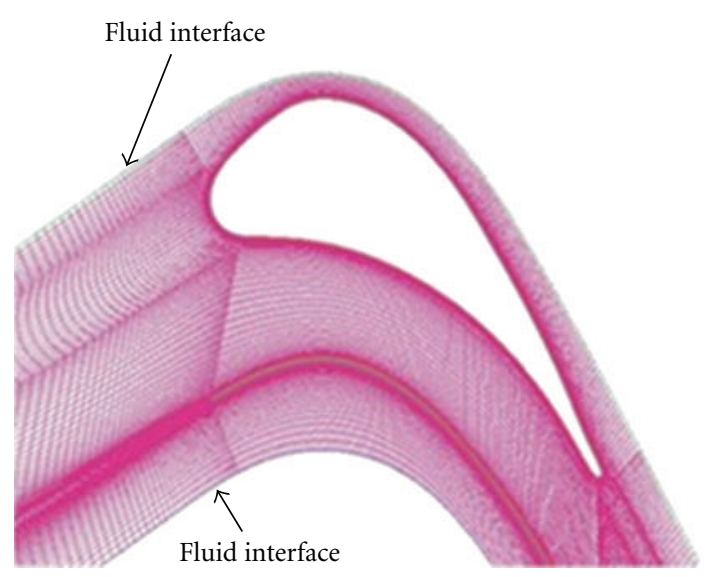

(a)

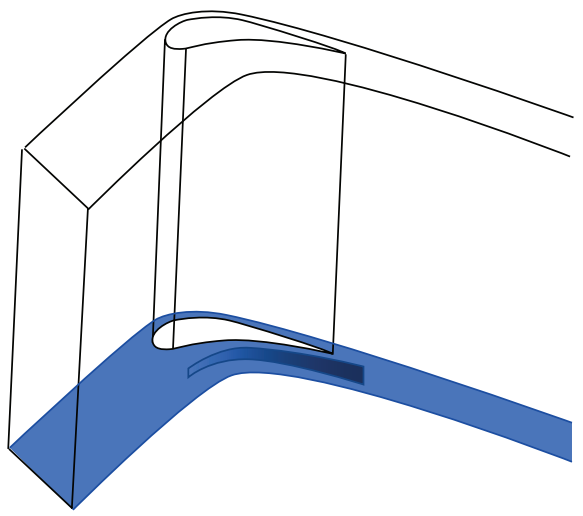

(b)

Figure 2: Typical mesh and a view of the computational domain with blade and fence.

no slip condition and are adiabatic. At inlet experimentally measured total pressure profile for $0^{\circ}$ incidence is specified with a turbulence intensity of $1 \%$ and integral length scale of $0.005 \mathrm{~m}$. Experimentally measured inlet boundary layer thickness $(\delta)$ of $0.04 \mathrm{~m}$ is used in present study, $(\delta / h=0.1)$. Outlet boundary condition is zero relative static pressure.

Computations are carried out for half the span height from the endwall by giving symmetry boundary condition for the midspan. Fluid interfaces are specified as periodic with nonconformal grids. ANSYS high-resolution discretization scheme was chosen for all calculations. For details regarding numerical scheme CFX manual [23] could be referred to. This scheme is second-order accurate in space but locally reduces to first-order accuracy near discontinuities in order to avoid nonphysical oscillations. There are 2.6 million hexahedral cells in the baseline case, and solution was found to be grid independent at this cell number (Figure 3). Values of $\overline{\overline{\mathrm{CSKE}}}$ show very small change after 1.9 million cells. At 2.6 million cells the changes in the values are quite small. Hence the solution was found to be grid independent at 2.6 million cells.

In the present investigations, SST [24] turbulence model is used along with the transition model. The transition model used for the present study is correlation based on Langtry et al. [25]. This model uses a blending function that allows switching between the $\kappa-\omega$ model in the sub and log layer and the $\kappa-\varepsilon$ model in the outer region of the boundary layer and in free-shear flows.

As the mesh is very fine near to the solid walls, low Reynolds number formulation of the turbulence model is employed instead of wall function approach. The solver runs and calculates the $y^{+}$value on walls. If the $y^{+}$value is greater than 3 , it will switch to wall function approach. If the $y^{+}$value is less than 3 , it will switch to near-wall model approach, that is, turbulence equations are solved near to the wall also. In all the test cases $y^{+}$value is less than 2 at all solid surfaces. Hence low Reynolds number formulation of turbulence model is used. The maximum $y^{+}$value for all the solid surfaces being less than 2 indicates that flow features near the wall are captured accurately. For the blade and fence

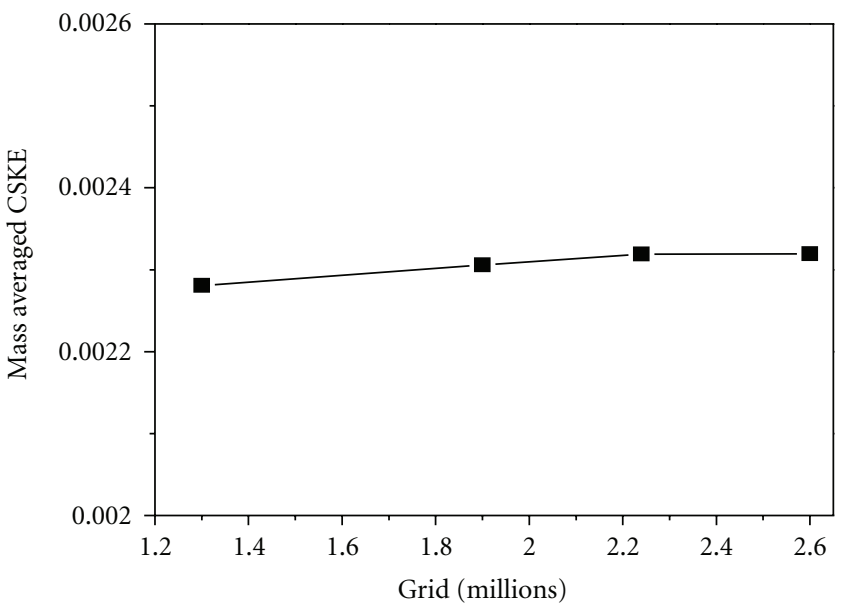

FIGURE 3: Variation of $\overline{\overline{\mathrm{CSKE}}}$ (baseline) at $x / a=1.06$ for different grids.

surfaces, cell height of $0.01 \mathrm{~mm}$ (first cell height normal to the surface) is used and contains 30 layers in a distance of $3 \mathrm{~mm}$ normal to the wall. For the endwall surface, cell height of $0.004 \mathrm{~mm}$ is used.

\section{Results and Discussion}

5.1. Validation. The exit measurements in computations as well as in experiments are taken at $x / a=1.06$. Figure 4 shows spanwise variation of pitchwise mass averaged exit flow angle for the baseline case. The agreement between computational and experimental exit angles is good except near the endwalls. The difference is $1^{\circ}$ in the midspan region. Near to the endwall overturning occurs, and away from it underturning occurs. CFD is predicting more overturning compared to the experimental measurements. The turning effect is due to the passage vortex which is dealt with in detail while discussing exit flow angle. Figure 5 shows the variation of pitchwise mass averaged nondimensional velocity ratio for the baseline case. Pitchwise and spanwise mass averaged 


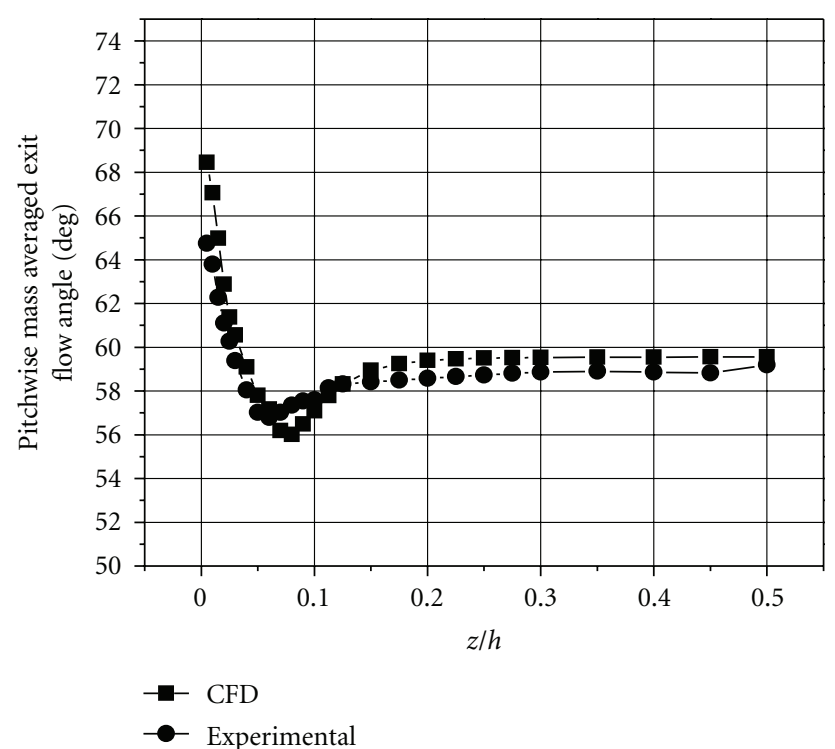

FIGURE 4: Spanwise variation of pitchwise mass averaged exit flow angle (baseline case) at $x / a=1.06$.

velocity at $x / a=1.06$ is used for nondimensionalising. The velocity ratios predicted by CFD are in excellent agreement with the measurements. Figure 6 shows the variation of pitchwise mass averaged CSKE. Figure 7 shows plot of pitchwise mass averaged exit flow angle variation with span for fence of $h_{f} / \delta=1 / 3$.

Despite using SST [24] model and maintaining the maximum $y^{+}$value below 2.0 for all the solid surfaces, the match between CFD and the experimental values of CSKE and exit flow angle is not satisfactory very near the endwall (Figures 4, 6, and 7). The possible reason for this discrepancy is deficiency in RANS (Reynolds Averaged Navier-Stokes) turbulence models. Quantitative prediction of the flow parameters near the endwall is not accurate because the flow here is vortex dominated and is in the boundary layer region. Most of the values of different correlation coefficients which are used in these turbulence models are derived from the wind tunnel experiments where the flow is made isotropic and homogeneous. But in a turbomachinery application due to the curvature of surfaces, the flow is neither isotropic nor homogeneous. Though SST-transition model showed improvements in results compared to other RANS models (Figures 14 and 15), the model is not able to address these effects completely.

In Figure 6 discrepancy between CFD and experimental values is high compared to those in Figures 4, 5, and Figure 7. It is because of error amplification in the computation of CSKE. Referring to (1), the expression for CSKE involves multiplication, addition, division, and then squaring. As per the rules of error analysis the error in the calculation of CSKE is amplified by 10 times. Because of above mentioned reasons the discrepancy in CSKE near the wall is very high. In Figures 4-7, CFD captures the trend near the endwall accurately. Away from the endwall, the match between CFD and experimental results is good.

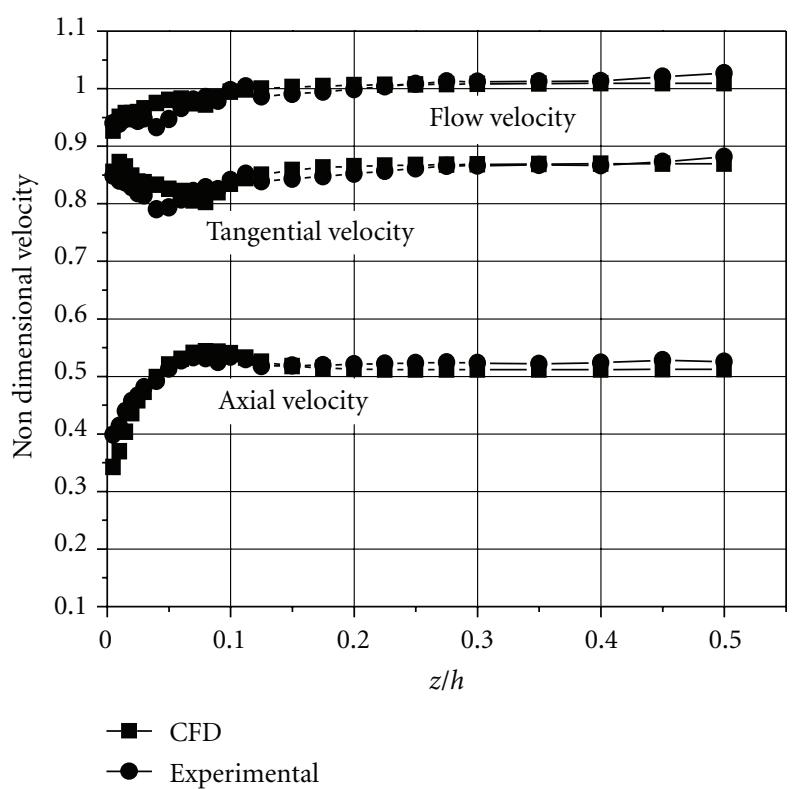

FIGURE 5: Spanwise variation of pitchwise mass averaged nondimensional velocity ratios (baseline case) at $x / a=1.06$.

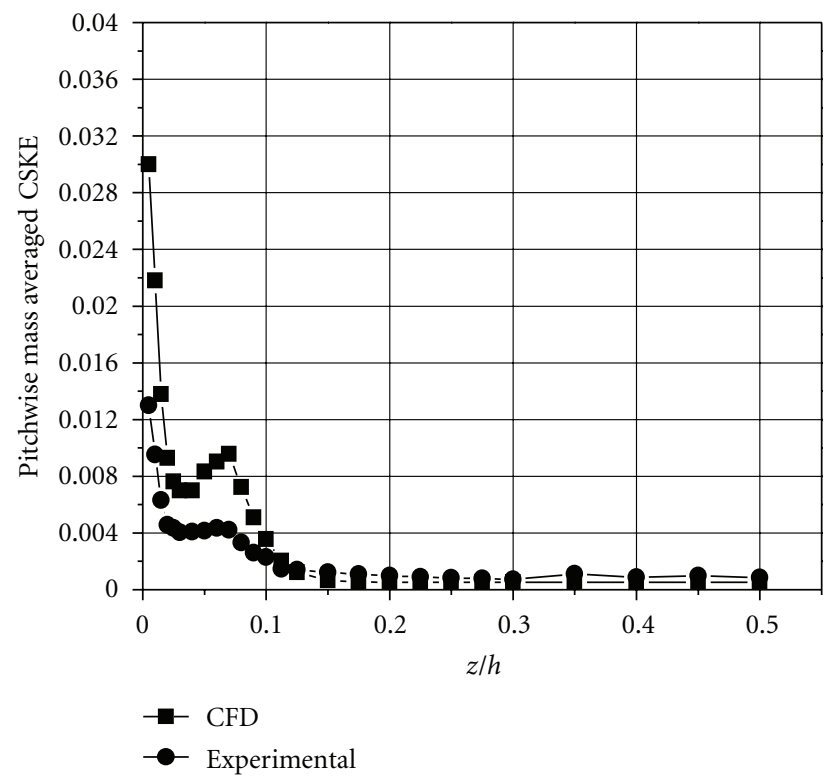

FIGURE 6: Spanwise variation of pitchwise mass averaged coefficient of secondary kinetic energy (baseline case) at $x / a=1.06$.

Comparison between computational and experimental results is also obtained for profile loss coefficient and is shown in Table 3. Profile loss coefficient, $Y_{P}$, is equal to the pitchwise mass averaged total pressure loss coefficient at midspan and is calculated from equation

$$
Y_{T}=\frac{\left(P_{01 \mathrm{MS}}-P_{02}\right)}{1 / 2 \rho \overline{\overline{C_{2}^{2}}}}
$$

where $Y_{T}$ is total pressure loss coefficient

$$
Y_{P}=\frac{\int Y_{T} c_{m} d y_{z / h=0.5}}{\int_{0}^{s} c_{m} d y}
$$




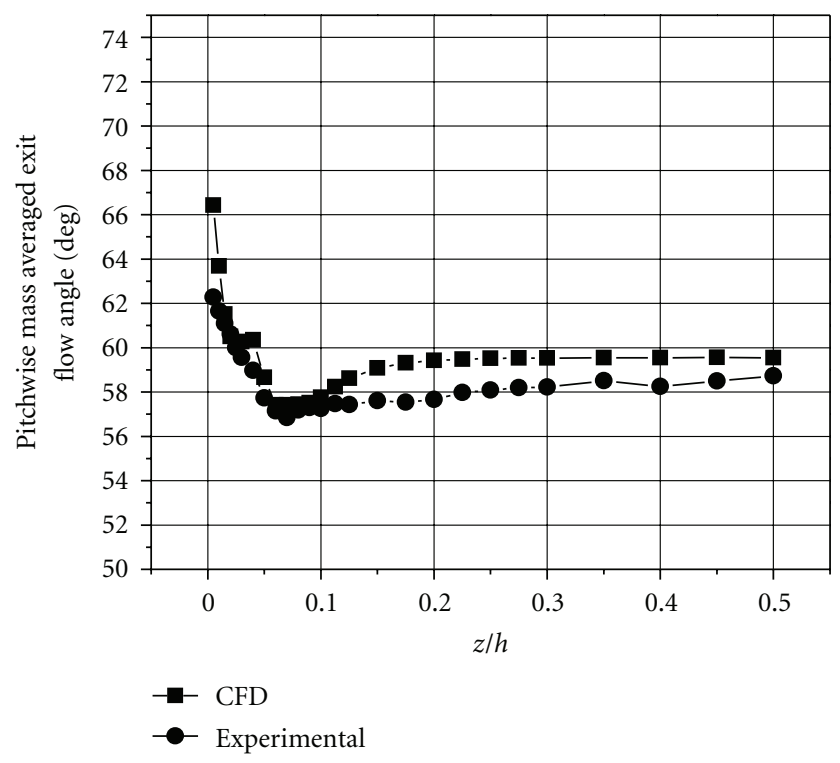

FIGURE 7: Spanwise variation of pitchwise mass averaged exit flow angle (fence of $h_{f} / \delta=1 / 3$ ) at $x / a=1.06$.

where $c_{m}$ is meridional velocity, $P_{01 \mathrm{MS}}$ is total pressure at midspan at inlet, $P_{02}$ is total pressure at $x / a=1.06$, and $z / h=0.5$ indicates midspan.

The difference between computed and experimental profile loss coefficient is $1.7 \%$ for the baseline case and $1 \%$ for fence of $h_{f} / \delta=1 / 3$. Close agreement between CFD and experimental values of profile loss coefficient shows that the numerical results are reasonable. At the midspan, SST turbulence model predicts reasonable result as the motion is nearly two-dimensional, free from vortices and has no endwall effect. Good prediction of profile loss in the present study is believed to be due to transition modelling and a very high-quality mesh. From Table 3 it can be seen that there is nearly no change in profile loss coefficient for the baseline case and fence of $h_{f} / \delta=1 / 3$ in experiment as well as in computation. Table 4 shows that experimental values of $\overline{\overline{\mathrm{CSKE}}}$ and $\overline{\overline{Y_{T}}}$ for fence of $h_{f} / \delta=1 / 3$ decrease compared to the baseline case. On the other hand computational investigation reports increase in $\overline{\overline{Y_{T}}}$ and decrease in $\overline{\overline{\mathrm{CSKE}}}$ for fence of $h_{f} / \delta=1 / 3$ compared to the baseline case. This confirms Langston [7] statement that total pressure loss prediction by CFD is not reasonable. The trend in the present investigations resembles the trend seen in Table 1 [8].

5.2. Optimum Fence. The fences were attached normal to the endwalls and were of the same camber line and the same stagger angle as the blades. The distance and the height of the fences were independently varied. Measurements of CSKE were done at $x / a=1.06$. Fence location from blade pressure surface is denoted by $y_{f} / s$, and fence height is denoted by $h_{f} / \delta$. When height of fence is varied linearly from $\delta / 6 \mathrm{~m}$ (at leading edge) to $\delta / 3 \mathrm{~m}$ (at trailing edge) and placed in middle, it produces least CSKE (Table 5). It is the optimum fence (in terms of height as well location) with a reduction of $27.8 \%$ CSKE with respect to the baseline case. The idea for this optimum fence came from the fact that as vortex travels downstream its size increases, hence keeping the height of the fence constant is not logical (Figure 8). As fence $\left(y_{f} / s=1 / 2\right.$, $\left.h_{f} / \delta=1 / 6\right)$ reduces CSKE by $15 \%$ and fence $\left(y_{f} / s=1 / 2\right.$, $h_{f} / \delta=1 / 3$ ) reduces CSKE by $17 \%$, therefore, it seems that $h_{f} / \delta=1 / 6$ is not sufficient along the entire streamwise direction. Hence it was decided to vary the height of the fence linearly from $h_{f} / \delta=1 / 6$ (at the leading edge) to $h_{f} / \delta=1 / 3$ (at the trailing edge).

Since CSKE is indicative of secondary flow losses, let us examine the role of CSKE in reducing the total pressure loss coefficient. The total pressure loss coefficient is given by

$$
\overline{\overline{Y_{T}}}=Y_{p}+Y_{s}+Y_{I} \text {. }
$$

The $Y_{I}$ is inlet loss coefficient and is calculated as

$$
\begin{gathered}
Y_{I N}=\left[\frac{p_{01 \mathrm{MS}}-p_{01}}{\rho / 2 \overline{\overline{c_{2}^{2}}}}\right], \\
Y_{I}=\frac{\int_{0}^{h / 2} \int_{0}^{s} Y_{I N} \overline{c_{m}} d y d z}{\int_{0}^{h / 2} \int_{0}^{s} \overline{c_{m}} d y d z},
\end{gathered}
$$

where $p_{01}$ is total pressure at $x / a=-1.5$ and $p_{01 \mathrm{MS}}$ is total pressure at midspan at $x / a=-1.5$.

In cases with streamwise endwall fences, it is observed that profile loss coefficient does not change much. Both experimental and numerical investigations indicate that there is negligible change in $Y_{P}$ (Table 3 ). Since calculation of $Y_{P}$ involves values at midspan where flow field is nearly two-dimensional and free from vortices, the prediction of $Y_{P}$ is accurate (Table 3). Table 6 shows the comparison of $Y_{P}$, $\overline{\overline{Y_{T}}}$, and $\overline{\overline{\mathrm{CSKE}}}$ for the optimum and baseline cases. In the present investigations inlet station is sufficiently away from the leading edge of blade ( $1.5 \mathrm{ch}$ length). Hence inlet loss coefficient $\left(Y_{I}\right)$ is same for the fenced and baseline cases.

The table also shows reduced $\overline{\overline{\mathrm{CSKE}}}$ values for the optimum fence case compared to the baseline case. On the other hand $Y_{T}$ value has increased over the baseline case which is a shortcoming with CFD approach. The results are in agreement with the results of Ingram [8] shown in Table 1. The total pressure loss coefficient from CFD is more than that obtained from experiments (Table 1). As prediction of $Y_{T}$ by CFD is not reasonable, the value of $Y_{T}$ (whether increasing or decreasing) is judged by its dependence on parameters which CFD predicts with reasonable accuracy. In the present investigations, the parameter chosen is CSKE. Since $\overline{\overline{\mathrm{CSKE}}}$ is indicative of secondary flow loss coefficient, $Y_{S}$, any reduction in $\overline{\overline{\mathrm{CSKE}}}$ is an indication of reduced $Y_{\mathrm{S}}$. As $Y_{P}$ and $Y_{I}$ are nearly constant, reduced $Y_{S}$ should result in reduced $\overline{\overline{Y_{T}}}$.

\subsection{Streamwise Vorticity Contours}

$$
\omega_{\mathrm{s}}=\omega_{\mathrm{x}} \cos \overline{\overline{\alpha_{2}}}+\omega_{\mathrm{y}} \sin \overline{\overline{\alpha_{2}}},
$$

where $\omega_{s}$ is streamwise vorticity, $\omega_{x}$ is vorticity in axial direction, and $\omega_{y}$ is vorticity in tangential direction. Nondimensional streamwise vorticity contours are plotted in 
TABle 3: Profile loss coefficient.

\begin{tabular}{|c|c|c|c|}
\hline \multicolumn{4}{|c|}{ Comparison of profile loss coefficients $\left(Y_{P}\right)$} \\
\hline & CFD & Experimental & $\%$ Difference \\
\hline Baseline case & 0.0334 & 0.0340 & 1.72 \\
\hline Fence of $h_{f} / \delta=1 / 3$ placed in middle of passage & 0.0337 & 0.0340 & 0.66 \\
\hline
\end{tabular}

TABLE 4: Comparison of $\overline{\overline{Y_{T}}}$ and $\overline{\overline{\mathrm{CSKE}}}$.

\begin{tabular}{lccc}
\hline & Baseline case & Fence of $h_{f} / \delta=1 / 3$ placed in the middle of passage & \% change w.r.t the baseline case \\
\hline$\overline{\overline{\text { CSKE}}}($ CFD $)$ & 0.0023 & 0.0019 & $-17 \%$ \\
$\overline{\overline{\text { CSKE }}}($ experimental $)$ & 0.0016 & 0.0011 & $-28 \%$ \\
$\overline{\overline{Y_{T}}}($ CFD $)$ & 0.0492 & 0.0519 & $5.5 \%$ \\
$\overline{\overline{Y_{T}}}($ experimental $)$ & 0.0751 & 0.0648 & $-14 \%$ \\
\hline
\end{tabular}

Figure 8 for test cases at $x / a=0.9$ (inside the passage) and at $x / a=1.06$ (outside the passage). Hatching in Figure 8 shows the sectional view of blade and fence at axial location $x / a=0.9$. In streamwise vorticity contours, passage vortex $(P)$ is the positive vortex. Negative streamwise vorticity is represented by dotted lines and positive streamwise vorticity by continuous lines. It can be seen that the magnitude of the passage vortex gets reduced as it goes downstream. This reduction in magnitude (vorticity) is caused by viscous action. It can be seen from Figures $8(\mathrm{c})$ and $8(\mathrm{~d})$ that the magnitude of the passage vortex near to the suction surface is reduced in the optimum fence case. This reduction in magnitude (by more than 50\%) and decrease in spanwise penetration of the passage vortex are due to the formation of counter rotating vortices (F, FI, and FII). Due to their opposite direction of rotation, these counter rotating vortices weaken the passage vortex $(P)$. Near to trailing edge, the flow on the blade pressure surface is towards the endwall, and on the suction surface it is towards the midspan. The flow from pressure and suction surfaces meets near the trailing edge and curls up to form trailing edge vortex $(T)$. Due to the presence of counter rotating vortex $(F)$ near the suction surface, the flow towards midspan on suction surface is decreased, resulting in weaker trailing edge vortices. The magnitude of trailing vortices $(T)$ is reduced by nearly $50 \%$. It is observed that as the height of fence increases the counterrotating vortex formed due to fence decreases and nearly vanishes after a fence of height $h_{f} / \delta=1.0$. Contours of all fence configurations cannot be presented here due to space limitation. From Table 5 it can be seen that for few fence heights and locations $\overline{\overline{\mathrm{CSKE}}}$ increases compared to the baseline case. It is because in these cases fences behave like blades and the passage vortex formed by them is comparable or in some cases even larger than the passage vortex formed by the actual blade. From Figures $8(\mathrm{e})$ and $8(\mathrm{f})$ it can be seen that fence of $h_{f} / \delta=4 / 3$ behaves like a blade of short span as it is producing vortex comparable to a normal blade. Passage vortices produced by fence of $h_{f} / \delta=4 / 3$ are located at higher spanwise locations and are of comparable magnitude to a normal blade. Because of this, three-dimensionality of the flow is increased and secondary kinetic energy is more for fence of $h_{f} / \delta=4 / 3$.

5.4. Flow Exit Angle. If the flow deflection is more than the geometrical deflection then flow overturning occurs, and if flow deflection is less compared to geometrical deflection then underturning occurs. The direction of rotation of passage vortex is such that, below its center (near to endwall), it increases the tangential velocity $\left(c_{\mathcal{u}}\right)$ of the fluid particles and above its center (away from endwall) the tangential velocity $\left(c_{u}\right)$ is reduced. Increase in $c_{u}$ increases exit flow angle and vice versa. Thus passage vortex induces flow overturning near to the endwall and flow underturning away from the endwall. The counter rotating vortex (F, FI, FII in Figures $8(\mathrm{c})$ and $8(\mathrm{~d})$ ) induces flow underturning near to endwall and flow overturning away from the endwall. This underturning of flow opposes the overturning of flow caused by passage vortex. Thus in optimum fence case overturning is reduced by more than $50 \%$ (compared to the baseline case) near to the endwall, which is evident from Figure 9. Underturning of flow away from the endwall is also reduced. Thus with the application of optimum fence, exit flow angle variation near to the endwall is reduced. These results are clear from Figure 10 which shows the contour plot of exit flow angle for the optimum fence and baseline cases. Near to the trailing edge (PS and SS, Figure 10) corner vortices near to the endwall are very strong in the baseline case, hence the flow has underturned remarkably by $11^{\circ}$. In case of optimum fence, corner vortices either seem to be of very low magnitude or were completely dissipated. As a result flow close to the endwall is overturned by $10^{\circ}$. These local values of exit flow angle in optimum fence case clearly show that effect of passage vortex has reduced considerably in optimum fence case compared to the baseline case.

5.5. Wall Shear. The inception and transport of the passage vortex and its impact on the surface boundary layers can be interpreted from the distribution of wall shear stress 


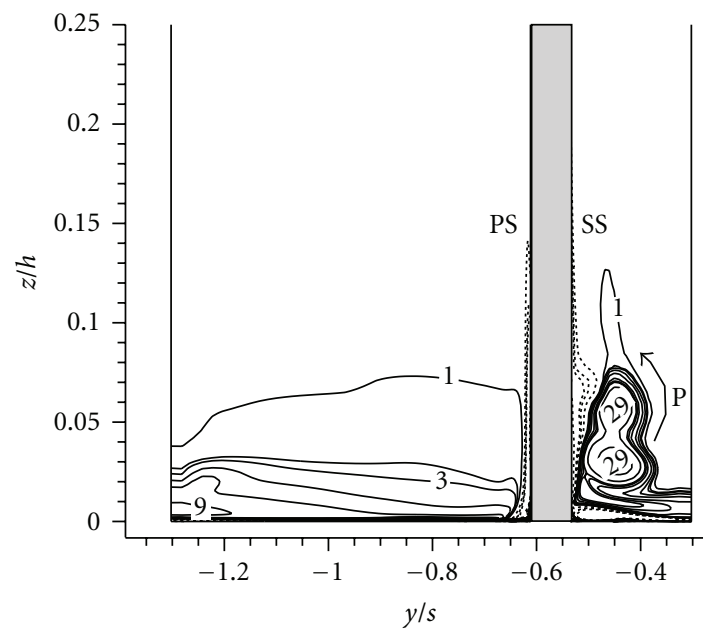

(a) Baseline at $x / a=0.9$

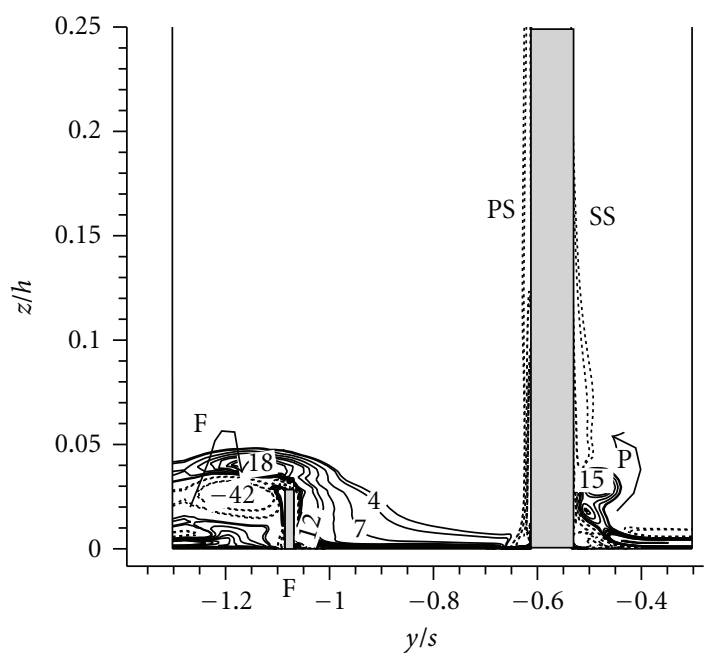

(c) With optimum fence at $x / a=0.9$

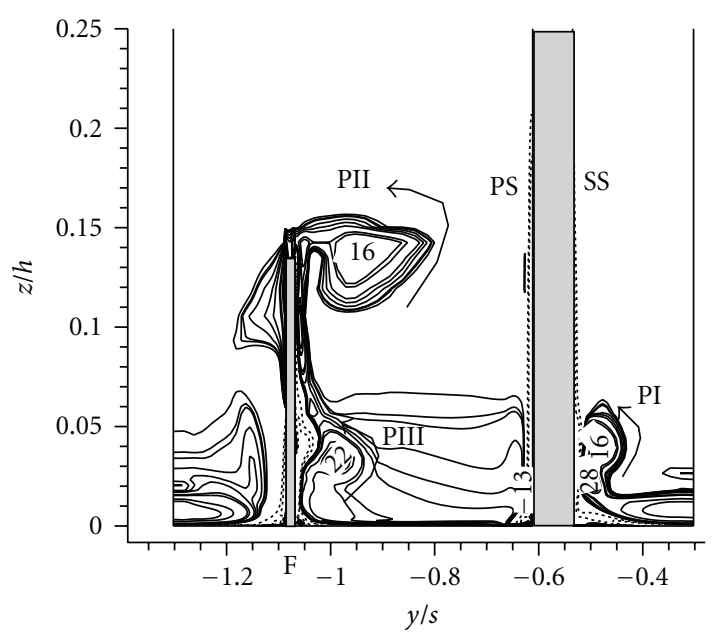

(e) With fence of $h_{f} / \delta=4 / 3$ at $x / a=0.9$

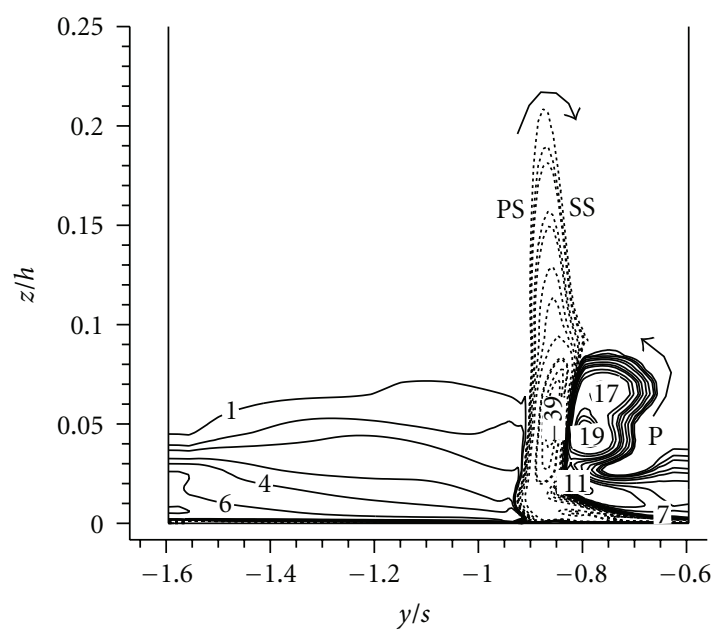

(b) Baseline at $x / a=1.06$

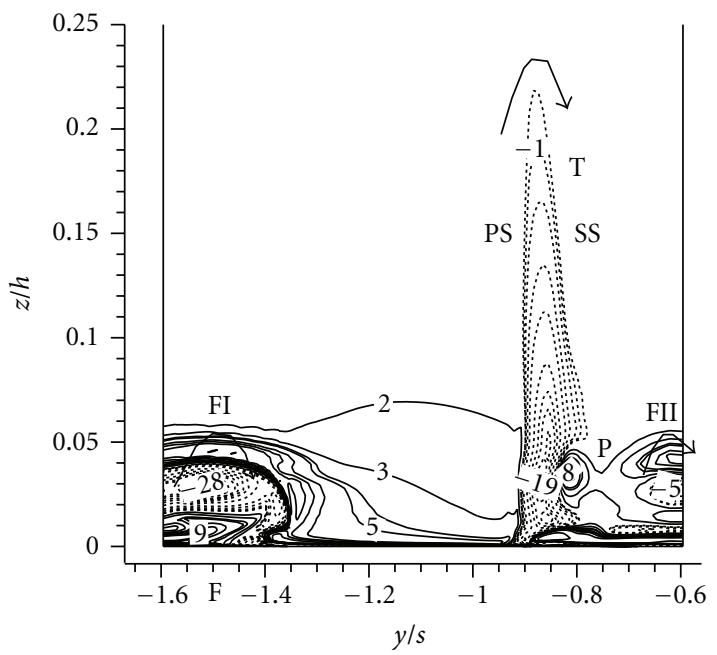

(d) With optimum fence at $x / a=1.06$

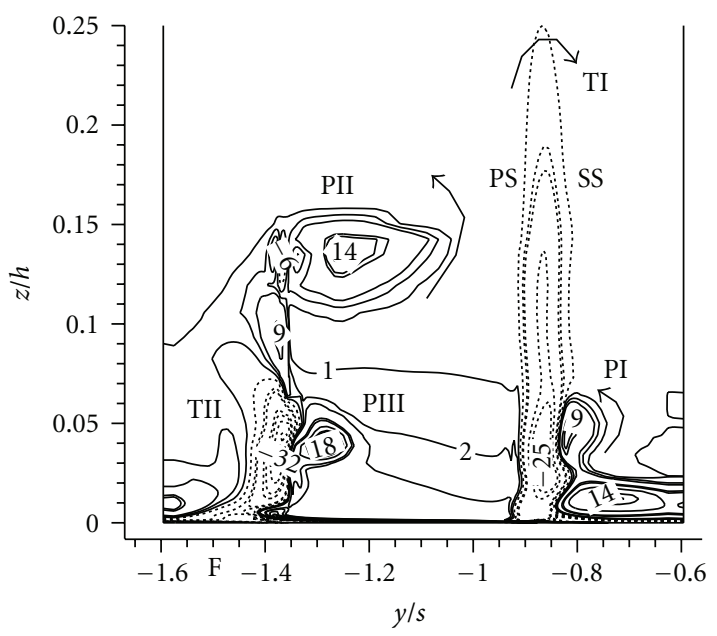

(f) With fence of $h_{f} / \delta=4 / 3$ at $x / a=1.06$

FIGURE 8: Contours of nondimensional streamwise vorticity $\left(c h \omega_{s} / \overline{\overline{c_{2}}}\right)$. 
TABLE 5: Mass averaged CSKE for fences of different height placed at different locations.

\begin{tabular}{lccc}
\hline S. no. & Fence location and fence height $\left(y_{f} / \mathrm{s}, h_{f} / \delta\right)$ & $\overline{\overline{\mathrm{CSKE}}}$ & \% reduction in $\overline{\overline{\mathrm{CSKE}}}$ \\
\hline 1 & $1 / 3,1 / 6$ & 0.002103 & -9.3 \\
2 & $\mathbf{1 / 2}, \mathbf{1 / 6}$ & $\mathbf{0 . 0 0 1 9 4 8}$ & $-\mathbf{1 5}$ \\
3 & $2 / 3,1 / 6$ & 0.002004 & -13.5 \\
4 & $1 / 3,1 / 3$ & 0.002035 & -12.3 \\
5 & $\mathbf{1 / 2 , 1 / 3}$ & $\mathbf{0 . 0 0 1 9 3 8}$ & $-\mathbf{1 7 . 0}$ \\
6 & $2 / 3,1 / 3$ & 0.001979 & -14.7 \\
7 & $1 / 2,2 / 5$ & 0.001955 & -15.7 \\
8 & $1 / 3,2 / 3$ & 0.00342 & 47.5 \\
9 & $1 / 2,2 / 3$ & 0.002031 & -12.4 \\
10 & $2 / 3,2 / 3$ & 0.001947 & -16.1 \\
11 & $1 / 3,1$ & 0.00513 & 121.2 \\
12 & $1 / 2,1$ & 0.002334 & 0.6 \\
13 & $2 / 3,1$ & 0.00254 & 9.5 \\
14 & $1 / 2,4 / 3$ & 0.002803 & -27.8 \\
15 & Baseline & $\mathbf{0 . 0 0 1 6 7 4}$ & - \\
\hline
\end{tabular}

TABLE 6: Comparison of $\overline{\overline{\mathrm{CSKE}}}, Y_{P}$ and $\overline{\overline{Y_{T}}}$.

\begin{tabular}{lccc}
\hline & Baseline case & Optimum fence & \% change w.r.t the baseline case \\
\hline$\overline{\overline{\mathrm{CSKE}}}(\mathrm{CFD})$ & 0.00231915 & 0.001674 & $-27.8 \%$ \\
$Y_{P}(\mathrm{CFD})$ & 0.033424 & 0.0334549 & $0.09 \%$ \\
$\overline{\overline{Y_{T}}}(\mathrm{CFD})$ & 0.04921 & 0.0507523 & $3.1 \%$ \\
\hline
\end{tabular}

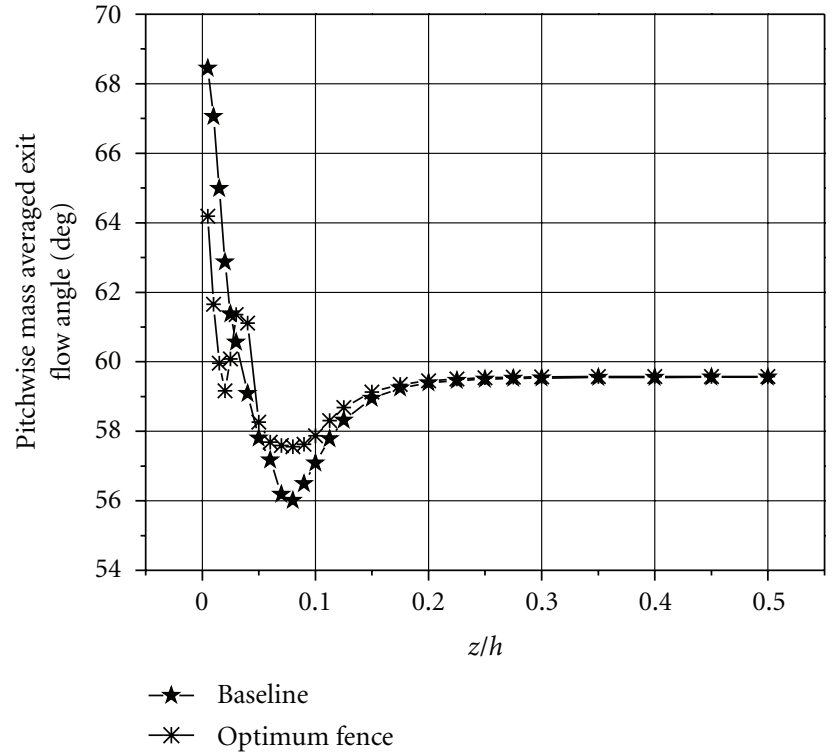

Figure 9: Pitchwise mass averaged exit flow angle for baseline and optimum fence cases at $x / a=1.06$.

and skin friction lines. Skin friction lines describe the flow immediately on the surface of a body. In a twodimensional flow, boundary layer separation is characterized by a reverse flow or vanishing wall shear stress, but in case of a three-dimensional flow vanishing shear is not the binding criterion. In three dimensional flow separations wall shear can have value other than zero. A necessary condition for the occurrence of three dimensional flow separation is the convergence of skin friction lines onto a separation line. The flow gets lifted along that separation line. Figure 11 shows contours of wall shear stress (with skin friction lines) on the suction surface for the baseline and optimum fence cases. On the suction surface there is formation of a separation bubble. The arrow of skin friction line shows the direction of fluid particle just above the surface. Flow separates (flow direction reverses) and reattaches in the separation bubble region. It could be seen in Figure 11 in separation bubble region that at point of separation the wall shear reduces to a very low value. The wall shear stress increases thereafter and then again decreases. Point of separation and point of reattachment are critical points, and hence the value of wall shear is zero. Critical points are points on surface of a body where wall shear value is zero and its direction is not defined at that point. The separation line shows the imprint of passage vortex on suction surface. $Z_{\mathrm{TE}}$ is defined as penetration height (from the endwall) of the passage vortex at the trailing edge on the airfoil suction surface. $Z_{\mathrm{TE}}$ in the baseline case is $0.029 \mathrm{~m}\left(Z_{\mathrm{TE}} / \delta=0.725\right)$, and in case of optimum fence it is $0.018 \mathrm{~m}\left(Z_{\mathrm{TE}} / \delta=0.45\right)$, Figure $11 \mathrm{~A}$ reduction of almost $33 \%$ is achieved in $Z_{\mathrm{TE}}$ by the use of optimum fence. According to Sharma and Butler [26], $Z_{\mathrm{TE}}$ 


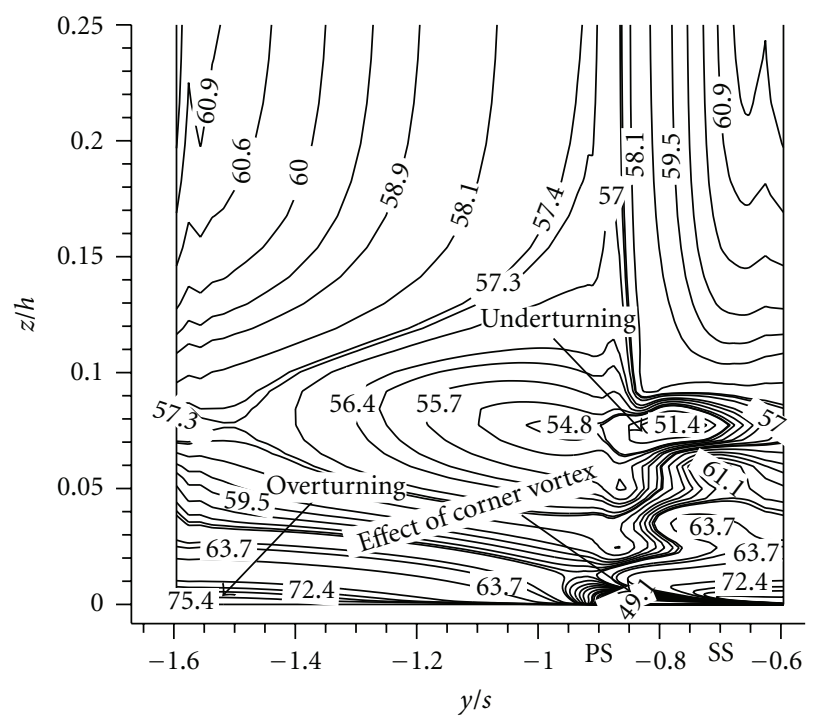

(a) Baseline

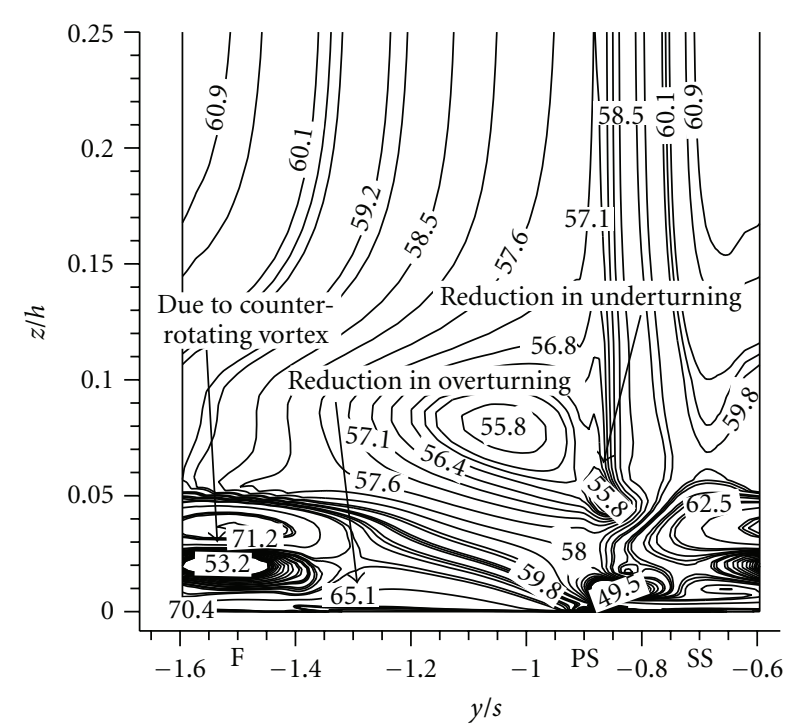

(b) Optimum fence

FIgURE 10: Contours of exit flow angle at $x / a=1.06$.

is indicative of secondary losses; hence optimum fence is effective in reducing secondary flow losses.

Figure 12 shows contours of wall shear (with skin friction lines) on the endwall surface. Near to the leading edge there is formation of a saddle point (Figure 12(b)). Saddle point is a critical point with zero wall shear. The separation lines are skin friction lines on which other skin friction lines converge. They are lift-off lines of the horseshoe and passage vortices. Separation lines are the footprint of these vortices. It could be seen from Figure 12(b) that one of the separation lines is blocked by fence whereas in the baseline case it goes and strikes the suction surface (Figure12(a)). The blocked leg of the vortex after striking the fence traverses along the suction surface and finally emerges from the passage. The separation line (the footprint of the pressure side leg of the horse shoe vortex) shows that after striking the fence the pressure side leg of the horse shoe vortex did not ride over the fence, rather it went downstream along the suction surface of the fence. Thus fence is instrumental in diverting the path of vortex. The shear stress is not low along the lift off line of the diverted vortex (Figures 12(a) and 12(b)). The wall shear along the separation line near to the suction surface of fence has an appreciable value compared to maximum wall shear value. Even then it lifts off from the surface. Hence it is not binding that separation will take place even if the wall shear stress is low.

5.6. Vortex Isosurface. Hunt [27] defined vortex as a region with positive second invariant $(Q>0)$ of gradient of velocity $(\nabla c)$. Physical interpretation of Hunt's definition is that vortex is a region where vorticity magnitude prevails over strain rate magnitude

$$
Q=-\frac{1}{2} u_{i, j} u_{i, j}=\frac{1}{2}\left(\|\Omega\|^{2}-\|S\|^{2}\right),
$$

where $\Omega$ and $S$ are rotation and strain tensors.

Figure 13 shows the isosurface of vortical structures for the optimum fence and baseline cases using $Q$ (second invariant of $\nabla c$ ). From isosurface visualization (Figure 13) it is visible that pressure side leg of the horseshoe vortex is diverted between pressure and suction side of the fence. From isosurface plot it can be concluded that optimum fence has trapped the pressure side leg of the horseshoe vortex.

5.7. Effect of Turbulence Model on $\overline{\overline{C S K E}}$ and Profile Loss Coefficient $\left(Y_{P}\right)$. Different turbulence models were used in the present investigations, and the quantitative difference in $\overline{\overline{\mathrm{CSKE}}}$ and $Y_{p}$ by different models is appreciable. Since experimental values for $\overline{\overline{\mathrm{CSKE}}}$ and $Y_{p}$ are available for fence of $h_{f} / \delta=1 / 3$, these are compared with the values obtained from the computations. Predicted values of CSKE and profile loss coefficient by $\kappa-\varepsilon$ models are the highest (Figures 14 and 15). The reason for this overprediction is the defect in the production term of turbulent kinetic energy equation. The production of turbulent kinetic energy in $\kappa$ $\varepsilon$ model is very high since the level of turbulence increases in the flow resulting in enhanced mixing. Enhanced mixing brings more losses and three-dimensionality in fluid. More three-dimensionality of flow results in higher CSKE values, and higher turbulence in boundary layer leads to more profile losses. Prediction of $\overline{\overline{\mathrm{CSKE}}}$ and $Y_{p}$ by SST model is lower compared to that of the $\kappa-\varepsilon$ model. This is due to the implementation of Kato-Launder modification and production limiters in the SST model. SST model coupled with transition model yields further lower values of $\overline{\overline{\mathrm{CSKE}}}$ and $Y_{p}$ because the boundary layer now is not completely turbulent.

The difference between CFD (SST transition) and experimental results is more in case of $\overline{\overline{\mathrm{CSKE}}}$ (Figure 14), and it is very less in case of profile loss coefficient (Figure 15). Profile loss coefficient involves measurements at midspan location, where turbulence models predict accurately; hence the agreement between CFD and experimental results is good. 


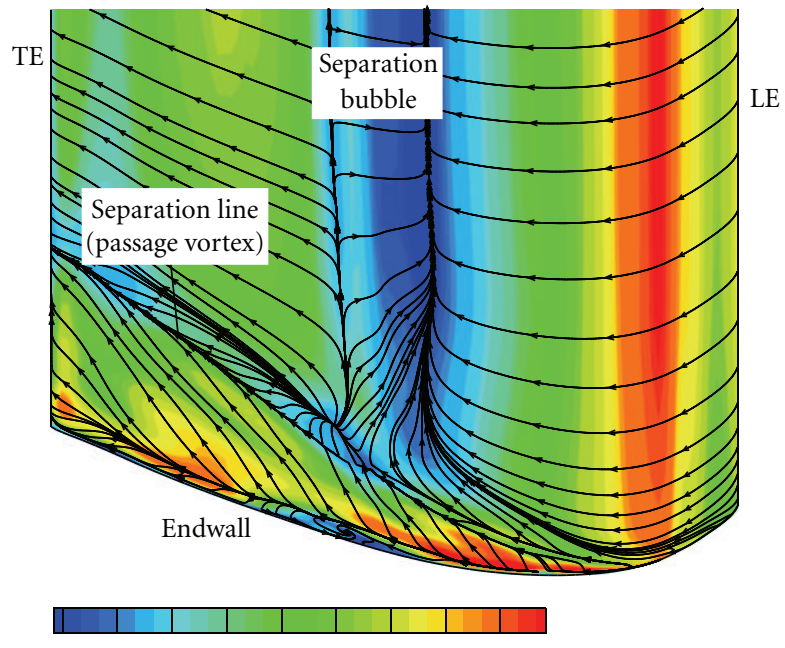

$\begin{array}{lllllllll}0.17 & 0.32 & 0.95 & 1.42 & 1.81 & 2.35 & 3.09 & 4.3 & 5.7\end{array}$

(a) Baseline case

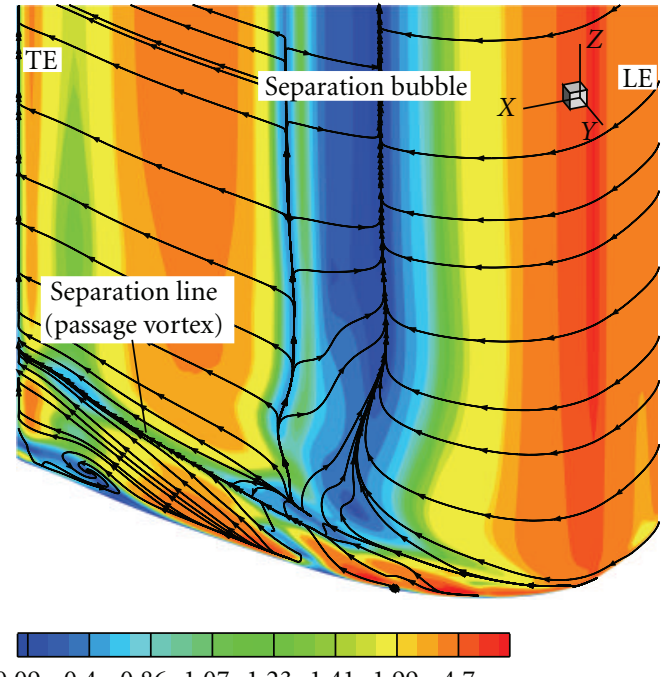

$\begin{array}{llllllll}0.09 & 0.4 & 0.86 & 1.07 & 1.23 & 1.41 & 1.99 & 4.7\end{array}$

(b) Optimum fence

FIGURE 11: Contours of wall shear stress on the suction surface (with skin friction lines).
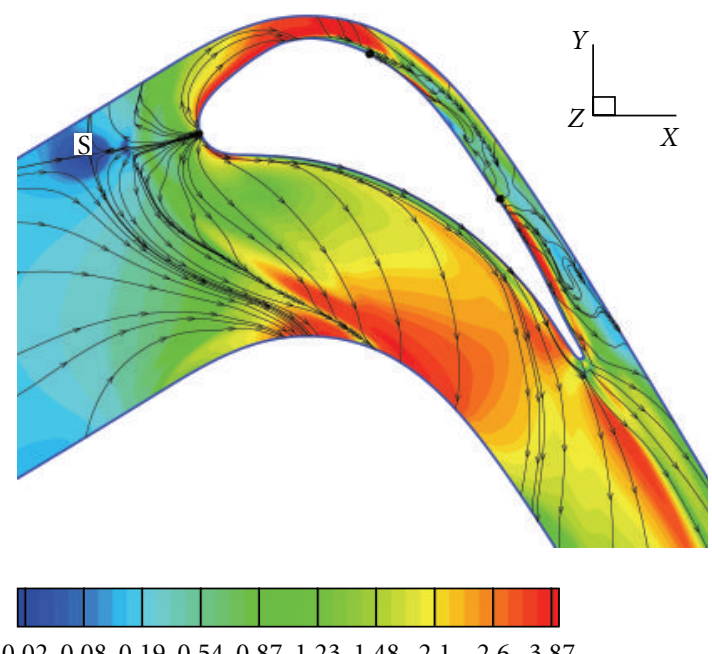

(a) Baseline case
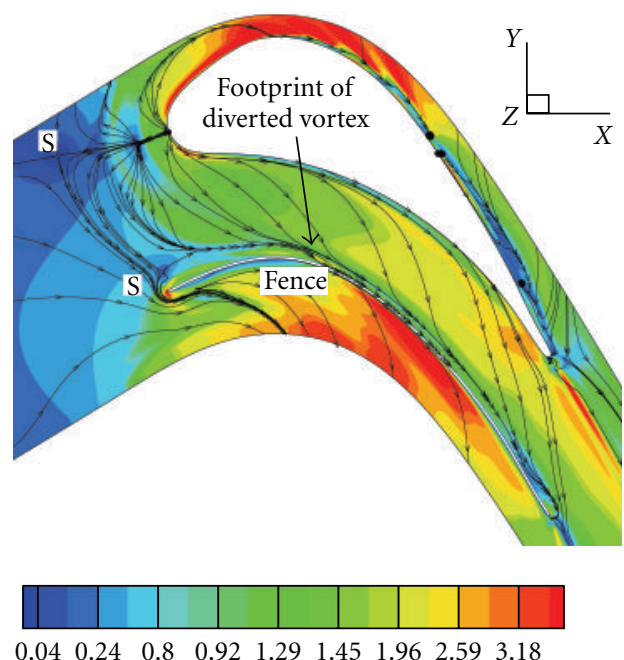

(b) Optimum fence

Figure 12: Contours of wall shear stress on the endwall (with skin friction lines).

$\overline{\overline{\mathrm{CSKE}}}$ involves values from midspan to the endwall. Near to the endwall, the prediction by turbulence models is only qualitatively true. Hence there is appreciable discrepancy between CFD (SST transition) and experimental results.

\subsection{Effectiveness of Optimum Fence at Enhanced Level of} Turbulence Intensity (Tu). In the present study, a range of engine relevant turbulence intensity levels is generated, and the resulting flow field is reported. This section assesses the effectiveness of fence at higher turbulence intensity levels. Higher turbulence intensity levels in gas turbines would typically increase the heat transfer rates on turbine blades and influence the boundary layer transition to a greater extent. On the suction surface of the airfoil the main effect of turbulence is to cause an earlier onset of transition to turbulent flow. From Figure 16 it is clear that even at high levels of turbulence intensity the optimum fence is producing less $\overline{\overline{\mathrm{CSKE}}}$, hence fence is effective even at enhanced levels of turbulence intensity. There is nearly no difference in profile loss coefficient for fenced and unfenced case (Figure 17), indicating that fence affects only endwall flow and not the midspan flow. When $\mathrm{Tu}$ is increased to $5 \%$, the size of the separation bubble diminishes resulting in lower profile loss. At further higher levels the boundary layer becomes completely turbulent resulting in more profile loss.

\subsection{Effectiveness of Optimum Fence at Off-Design Incidences.} If flow incidence is positive there will be increase in net flow deflection resulting in higher change in momentum, leading to increase in lift or blade loading. Similarly if flow 


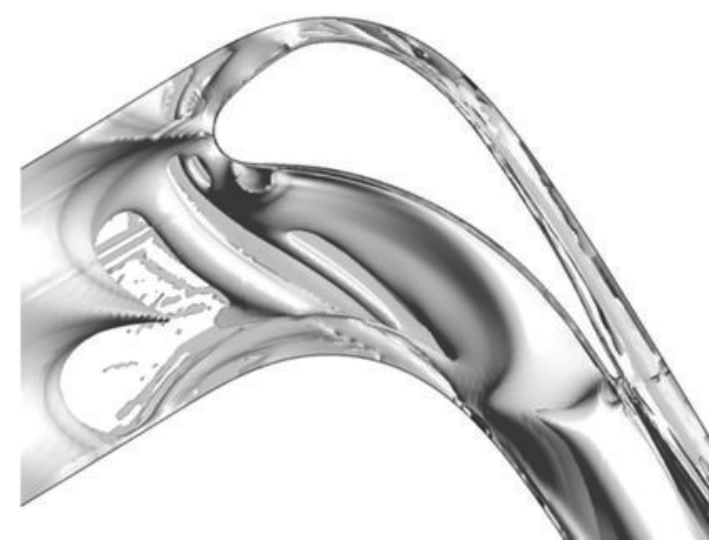

(a) Baseline case

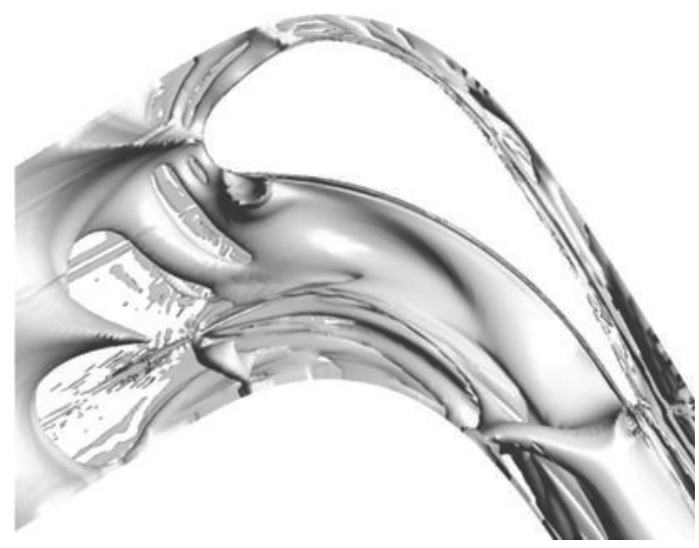

(b) Optimum fence

Figure 13: Vortex isosurface using $Q=0.01$.

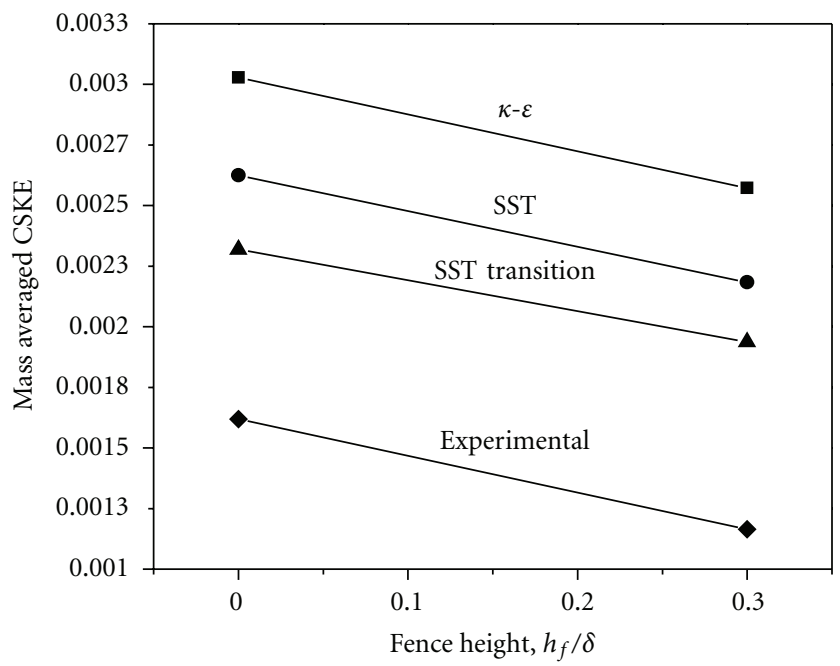

FIgURE 14: Variation of CSKE (at $x / a=1.06$ ) for different turbulence models.

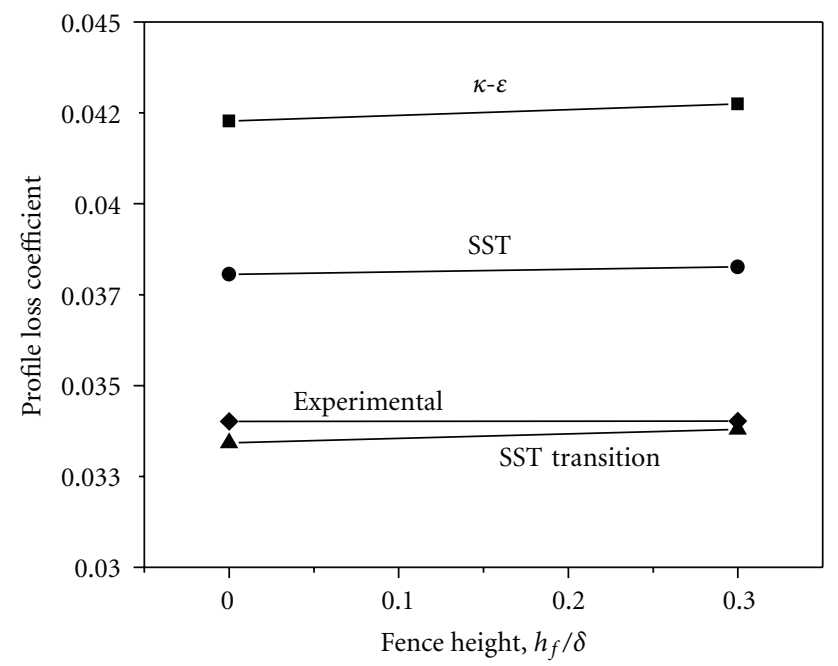

Figure 15: Variation of Profile loss coefficient (at $x / a=1.06$ ) for different turbulence models.

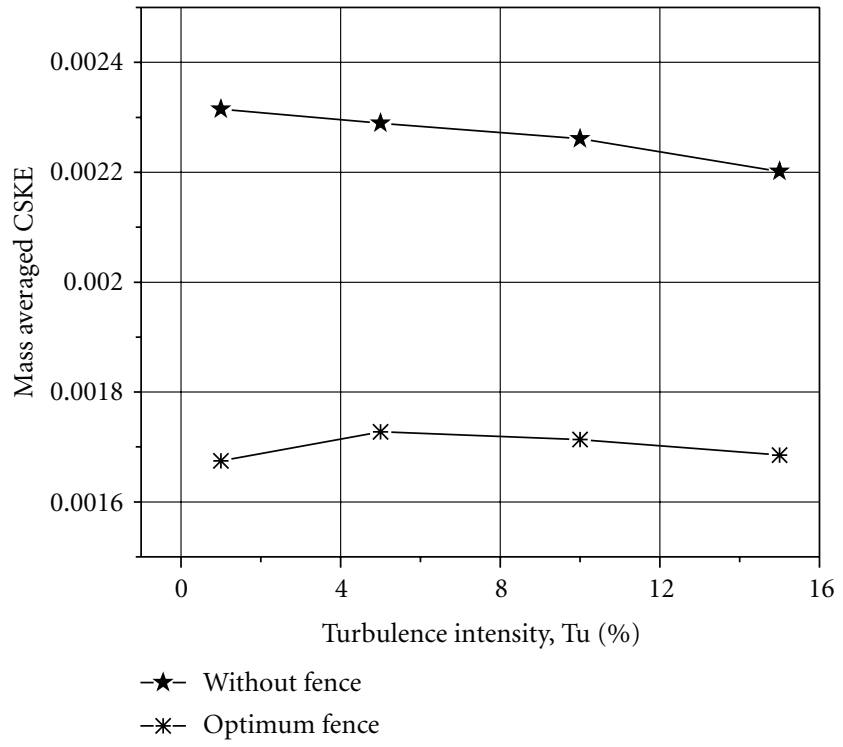

FIGURE 16: Variation of mass averaged CSKE at different turbulence intensity for optimum fence and unfenced case.

incidence is negative, net flow deflection will be less leading to reduction in blade loading. Secondary flow in a turbine is dependent on net flow deflection of flow. This can be concluded from the transport vorticity equation which is obtained by forming the curl of the Navier-Stokes equation. In an intrinsic coordinate system the streamwise component can be written as follows:

$$
\frac{D \omega_{s}}{D t}=\omega_{s} \frac{\partial u}{\partial s}+\omega_{n} \frac{\partial u}{\partial n}+\omega_{z} \frac{\partial u}{\partial z}+\nu \nabla^{2} \omega_{s} .
$$

If the viscous term is neglected, it follows that in the entrance region of a linear turbine cascade the rate of change of streamwise vorticity $\left(\omega_{s}\right)$ depends only on the normal vorticity $\left(\omega_{n}\right)$ (due to the inlet boundary layer) and on the velocity gradient $\partial u / \partial n$ in the normal direction, as $\left(\omega_{s}\right)$ and $\left(\omega_{z}\right)$ are zero at the inlet. $\partial u / \partial n$ increases with the blade loading. Therefore, when the incidence angle is increased 


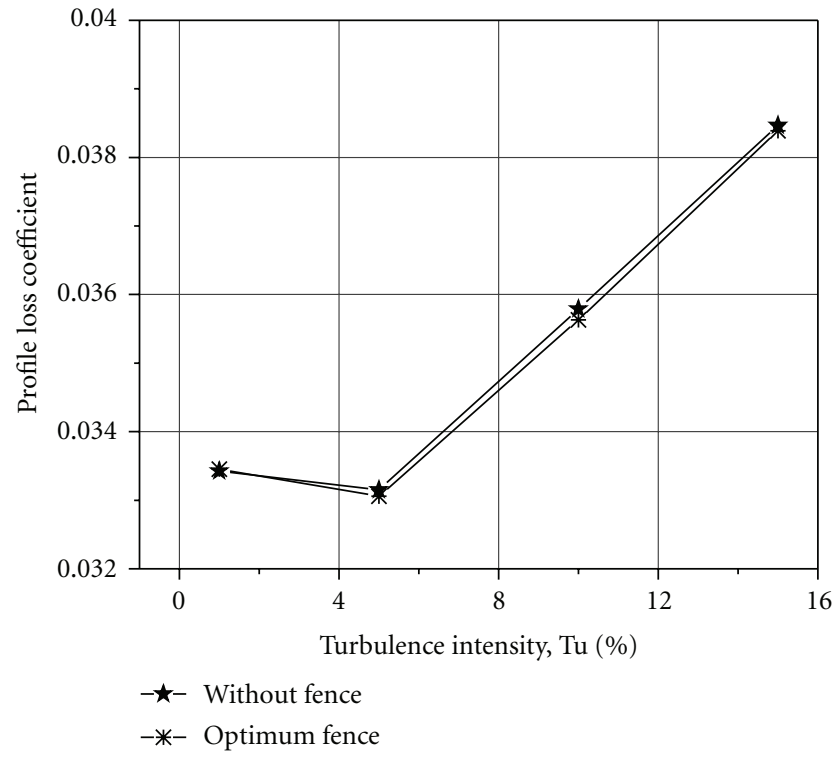

Figure 17: Variation of profile loss coefficient at different turbulence intensities for optimum and baseline cases.

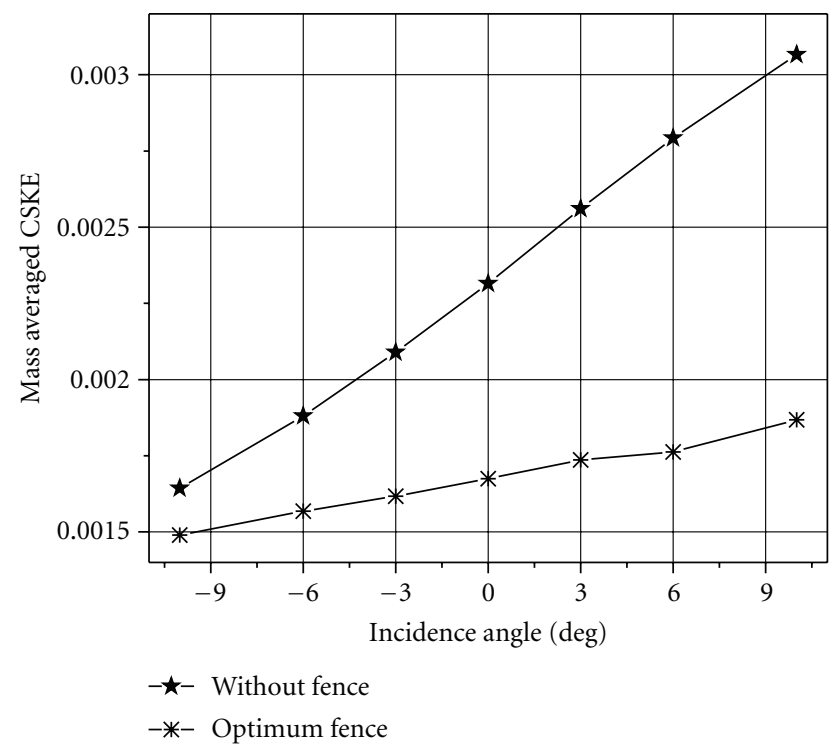

FIGURE 18: Variation of $\overline{\overline{\mathrm{CSKE}}}$ with incidence angle for the optimum and baseline cases.

higher secondary flow effects must be expected due to larger velocity gradients between pressure and suction surfaces. Similarly when the incidence angle is decreased, reduced secondary flow effects must be expected. Streamwise vorticity $\left(\omega_{s}\right)$ represents secondary flow. For the above mentioned reason, $\overline{\overline{\mathrm{CSKE}}}$ increases at positive incidence angles and decreases at negative incidence angles (Figure 18).

From Figure 18 it is clear that even at off-design incidence angles the optimum fence generates less $\overline{\overline{\mathrm{CSKE}}}$ compared to unfenced case. There is no appreciable difference in profile loss coefficient between two cases (Figure 19). As both fenced and baseline cases have same inlet conditions, they will have

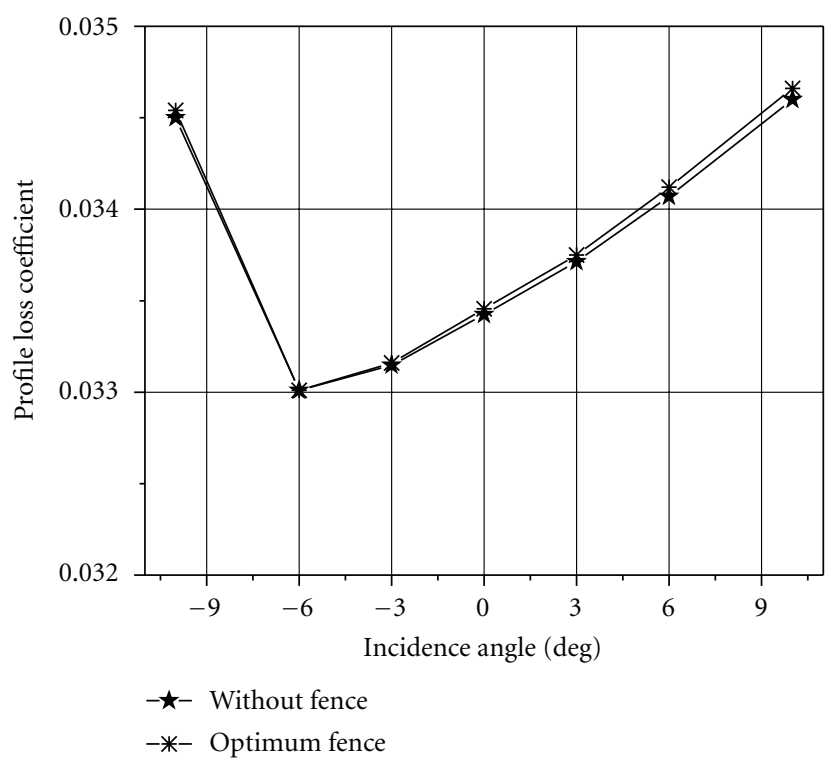

FIGURE 19: Variation of profile loss coefficient with incidence angle for the optimum and baseline cases.

same inlet loss coefficient. From (5) it can be concluded that at off design incidence angles the optimum fence will have lesser total pressure loss coefficient compared to baseline case.

\section{Conclusions}

A fence whose height varies linearly from $\delta / 6$ at the leading edge to $\delta / 3$ at the trailing edge and located in the middle of the passage produces least $\overline{\overline{\mathrm{CSKE}}}$ and is determined to be the optimum fence. Design of such optimum fence is completely new and reported for the first time. Optimum fence has reduced $\overline{\overline{\mathrm{CSKE}}}$ by $27 \%$ compared to $17 \%$ for fence of $h_{f} / \delta=1 / 3$. The optimum fence showed reduction in overturning and underturning of flow by nearly $50 \%$. Reduction in the exit flow angle deviation will reduce the incidence losses in the next stage of turbine. Hence there is scope for improvement in turbine stage efficiency as well. The magnitude and spanwise penetration of passage vortex $\left(Z_{\mathrm{TE}}\right)$ for the optimum fence is reduced by $33 \%$ compared to the baseline case. As $Z_{\mathrm{TE}}$ is an indicative of secondary flow losses, optimum fence is effective in reducing secondary flow losses. It is observed that optimum fence does not change the midspan flow field, and therefore there is nearly no change in profile loss coefficient. Optimum fence is effective even at higher turbulence levels and off design incidences. These observations infer that the optimum fence is instrumental in reducing the secondary flow effects in a turbine cascade.

\section{Nomenclature}

a: Axial chord $(\mathrm{m})$

c: Velocity $(\mathrm{m} / \mathrm{s})$

ch: Blade chord (m) 
$c_{m}: \quad$ Axial velocity $(\mathrm{m} / \mathrm{s})$

$c_{r}: \quad$ Radial velocity $(\mathrm{m} / \mathrm{s})$

$c_{u}$ : Tangential velocity $(\mathrm{m} / \mathrm{s})$

CSKE: Coefficient of secondary kinetic energy

$h_{f}$ : Fence height $(\mathrm{m})$

LE: Leading edge

$P: \quad$ Passage vortex

p: $\quad$ Pressure $\left(\mathrm{N} / \mathrm{m}^{2}\right)$

S: $\quad$ Strain tensor $\left(\mathrm{s}^{-1}\right)$

$t: \quad$ Fence thickness $(\mathrm{m})$

$T$ : $\quad$ Trailing vortex

TE: Trailing edge

Tu: Turbulence intensity

$Y_{I}: \quad$ Inlet loss coefficient

$y_{f}: \quad$ Distance from the pressure surface $(\mathrm{m})$

$Y_{p}: \quad$ Profile loss coefficient

$Y_{T}$ : Total pressure loss coefficient.

\section{Subscripts}

1,2: $\quad$ Cascade inlet and outlet

$x, y, z, s$ : Components in axial, pitchwise, spanwise, and streamwise directions

$i, j: \quad$ Index notations $(1,2,3)$.

\section{Superscripts}

-: Pitchwise mass averaged quantity

$=$ : Pitchwise and spanwise mass averaged quantity.

\section{Greek Symbols}

$\alpha$ : Flow angle measured from the axial direction (deg)

$\delta$ : Inlet boundary layer thickness (m)

$\tau$ : Tip gap height $(\mathrm{m})$

$\omega$ : Vorticity $\left(\mathrm{s}^{-1}\right)$

$\Omega$ : Rotation tensor $\left(\mathrm{s}^{-1}\right)$.

\section{References}

[1] T. Kawai, "Effect of combined boundary layer fences on turbine secondary flow and losses," JSME International Journal, Series B, vol. 37, no. 2, pp. 377-384, 1994.

[2] Y. J. Moon and S. R. Koh, "Counter-rotating streamwise vortex formation in the turbine cascade with endwall fence," Computers and Fluids, vol. 30, no. 4, pp. 473-490, 2001.

[3] M. Govardhan, A. Rajender, and J. P. Umang, "Effect of streamwise fences on secondary flows and losses in a twodimensional turbine rotor cascade," Journal of Thermal Science, vol. 15, no. 4, pp. 296-305, 2006.

[4] C. Camci and D. H. Rizzo, "Secondary flow and forced convection heat transfer near endwall boundary layer fences in a $90^{\circ}$ turning duct," International Journal of Heat and Mass Transfer, vol. 45, no. 4, pp. 831-843, 2002.

[5] J. T. Chung, T. W. Simon, and J. Buddhavarapu, "Threedimensional flow near the blade/endwall junction of a gas turbine: application of a boundary layer fence," in Proceedings of the 36th ASME International Gas Turbine and Aeroengine
Congress and Exposition, Orlando, Fla, USA, 1991, ASME paper no. 91-GT-45.

[6] J. T. Chung and T. W. Simon, "Effectiveness of the gas turbine endwall fences in secondary flow control at elevated free-stream turbulence levels," in Proceedings of the 38th ASME International Gas Turbine and Aeroengine Congress and Exposition, 1993, ASME paper no. 93-GT-51.

[7] L. S. Langston, "Secondary flows in axial turbines-a review, heat transfer in gas turbine systems," Annals of the New York Academy of Sciences, vol. 934, pp. 11-26, 2001.

[8] G. L. Ingram, End Wall Profiling for the Reduction of Secondary Flow in Turbines, Ph.D. thesis, University of Durham, 2003.

[9] G. Ingram, D. G. Gregory-Smith, and N. W. Harvey, "Investigation of a novel secondary flow feature in a turbine cascade with end wall profiling," ASME Journal of Turbomachinery, vol. 127, no. 1, pp. 209-214, 2005.

[10] M. Rose, N. Harvey, P. Seaman, D. Newman, and D. McManus, "HP turbine using non-axisymmetric end walls: part IIexperimental validation," in Proceedings of the American Society of Mechanical Engineers, 2001, ASME paper no. 2001GT-0505.

[11] N. W. Harvey, G. Brennan, D. A. Newman, and M. G. Rose, "Improving turbine efficiency using non-axisymmetric end walls: validation in the multi-row environment and with low aspect ratio blading," in Proceedings of the American Society of Mechanical Engineers Turbo Expo, pp. 119-126, 2002, ASME paper no. GT-2002-30337.

[12] J. C. Hartland, D. G. Gregory-Smith, N. W. Harvey, and M. G. Rose, "Nonaxisymmetric turbine end wall design: part IIexperimental validation," ASME Journal of Turbomachinery, vol. 122, no. 2, pp. 286-293, 2000.

[13] M. W. Benner, S. A. Sjolander, and S. H. Moustapha, "An empirical prediction method for secondary losses in turbines: part II-a new secondary loss correlation," ASME Journal of Turbomachinery, vol. 128, no. 2, pp. 281-291, 2006.

[14] R. Corral and F. Gisbert, "Profiled end wall design using an adjoint Navier-Stokes solver," ASME Journal of Turbomachinery, vol. 130, no. 2, Article ID 021011, 2008.

[15] G. Brennan, N. W. Harvey, M. G. Rose, N. Fomison, and M. D. Taylor, "Improving the efficiency of the trent 500-HP turbine using nonaxisymmetric end walls: part I-turbine design," ASME Journal of Turbomachinery, vol. 125, no. 3, pp. 497-504, 2003.

[16] D. A. Bagshaw, G. L. Ingram, D. G. Gregory-Smith, and M. R. Stokes, "An experimental study of three-dimensional turbine blades combined with profiled endwalls," Proceedings of the Institution of Mechanical Engineers, Part A: Journal of Power and Energy, vol. 222, no. 1, pp. 103-110, 2008.

[17] M. G. Nagel and R. D. Baier, "Experimentally verified numerical optimization of a three-dimensional parametrized turbine vane with nonaxisymmetric end walls," ASME Journal of Turbomachinery, vol. 127, no. 2, pp. 380-387, 2005.

[18] A. Duden, I. Raab, and L. Fottner, "Controlling the secondary flow in a turbine cascade by three-dimensional airfoil design and endwall contouring," ASME Journal of Turbomachinery, vol. 121, no. 2, pp. 191-199, 1999.

[19] N. W. Harvey, M. G. Rose, S. Shahpar, M. D. Taylor, J. Hartland, and D. G. Gregory-Smith, "Nonaxisymmetric turbine end wall design: part I-three-dimensional linear design system," ASME Journal of Turbomachinery, vol. 122, no. 2, pp. 278-285, 2000.

[20] T. J. Pralsner, E. Allen-Bradley, E. A. Grover, D. C. Knezevici, and S. A. Sjolander, "Application of non-axisymmetric endwall contouring to conventional and high-lift turbine airfoils," 
in Proceedings of the ASME Turbo Expo, 2007, ASME paper no. GT-2007-27579.

[21] T. Germain, M. Nagel, I. Raab, P. Schupbach, R. S. Abhari, and M. Rose, "Improving efficiency of a high work turbine using nonaxisymmetric endwalls: part I-endwall design and performance," ASME Journal of Turbomachinery, vol. 132, no. 2, Article ID 021007, pp. 1-9, 2010.

[22] P. Schüpbach, M. Rose, J. Gier, I. Raab, T. Germain, and R. S. Abhari, "Non-axisymmetric endwall profiles including fillet radii, in a 1.5 stage axial flow turbine," in Proceedings of the 8 th European Conference on Turbomachinery Fluid Dynamics and Thermodynamics (ETC '09), Graz, Austria, 2009.

[23] ANSYS CFX 11.0 Manual, 2009.

[24] F. R. Menter, "Two-equation eddy-viscosity turbulence models for engineering applications," AIAA Journal, vol. 32, no. 8, pp. 1598-1605, 1994.

[25] R. B. Langtry, F. R. Menter, S. R. Likki, Y. B. Suzen, P. G. Huang, and S. A. Völker, "A correlation-based transition model using local variables - part II: test cases and industrial applications," ASME Journal of Turbomachinery, vol. 128, no. 3, pp. 423-434, 2006.

[26] O. P. Sharma and T. L. Butler, "Prediction of endwall losses and secondary flows in axial flow turbine cascades," ASME Journal of Turbomachinery, vol. 109, no. 2, pp. 229-236, 1987.

[27] J. Jeong and F. Hussain, "On the identification of a vortex," Journal of Fluid Mechanics, vol. 285, pp. 69-94, 1995. 

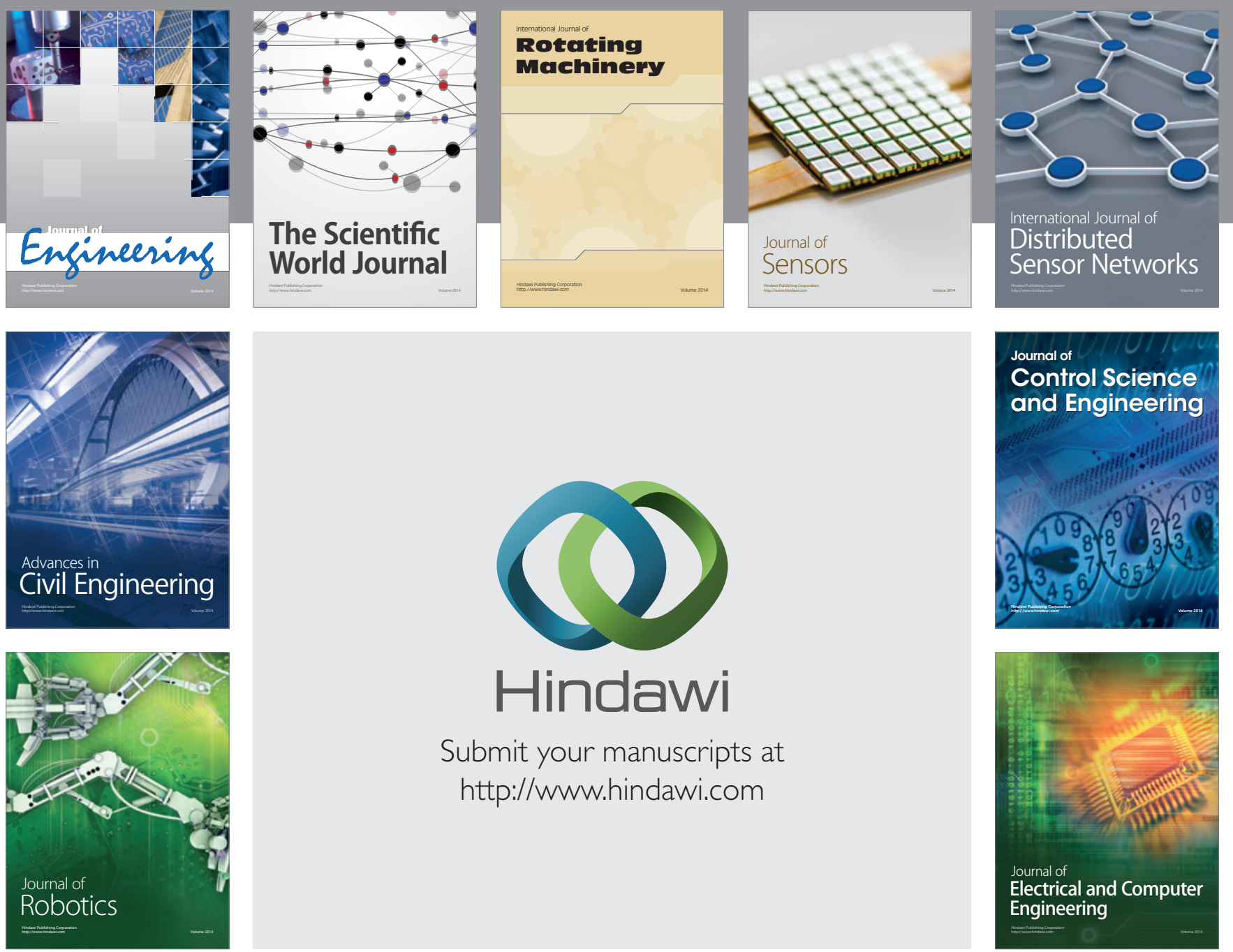

Submit your manuscripts at

http://www.hindawi.com
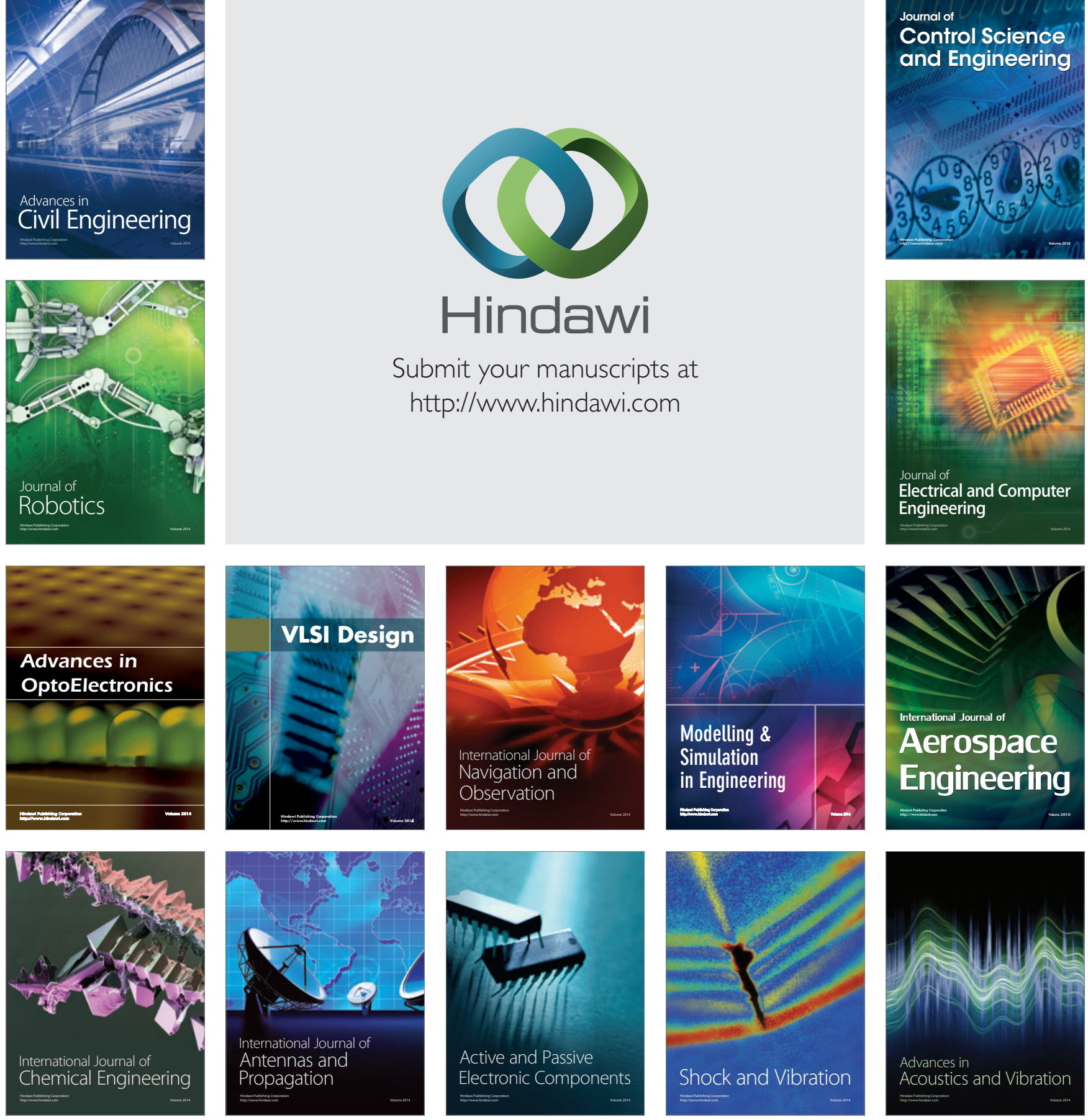